\title{
On active disturbance rejection control for lower- triangular nonlinear uncertain systems with mismatched uncertainties and unknown control coefficients
}

Ping Liu

Sun Yat-Sen University

Sen Chen ( $\square$ chensen14@mails.ucas.ac.cn )

Shaanxi Normal University

Zhi-Liang Zhao

Shaanxi Normal University

\section{Research Article}

Keywords: nonlinear uncertain system, active disturbance rejection control, mismatched uncertainty, unknown control coefficient

Posted Date: May 17th, 2021

DOl: https://doi.org/10.21203/rs.3.rs-507905/v1

License: (c) (1) This work is licensed under a Creative Commons Attribution 4.0 International License.

Read Full License 


\title{
On active disturbance rejection control for lower-triangular nonlinear uncertain systems with mismatched uncertainties and unknown control coefficients
}

\author{
Ping Liu ${ }^{\mathrm{a}}$, Sen Chen ${ }^{\mathrm{b}, *}$, Zhi-Liang Zhao ${ }^{\mathrm{b}}$ \\ ${ }^{a}$ School of Aeronautics and Astronautics, Sun Yat-sen University, Guangdong 510275, China; \\ ${ }^{b}$ School of Mathematics and Information Science, Shaanxi Normal University, Xi'an 700190, China.
}

\begin{abstract}
The paper investigates the control problem for a class of lower-triangular nonlinear uncertain systems with mismatched uncertainties and unknown values of control coefficients. Based on the signs of control coefficients rather than the nominal values or the approximative mathematical expressions, a new active disturbance rejection control is proposed. The design procedure can be concluded by three steps: determining the equivalent integrators chain form, constructing the extended state observer to estimate the total disturbance, and designing a dynamical system to let the actual input track the ideal input. Then under a mild assumption for mismatched uncertainties and unknown control coefficients, the paper rigorously analyzes the bounds of tracking error, estimating error and the error between the actual and ideal inputs. The presented theoretical results reveal the strong robustness of the proposed method to mismatched uncertainties and uncertain control input coefficients. Moreover, the tuning law of observer parameter and the parameter of dynamical input design is theoretically shown.

Keywords: nonlinear uncertain system, active disturbance rejection control, mismatched uncertainty, unknown control coefficient.
\end{abstract}

\footnotetext{
${ }^{*}$ Corresponding author

Email addresses: liup89@mail.sysu. edu.cn (Ping Liu),

chensen14@mails.ucas.ac.cn (Sen Chen), zhiliangzhao@snnu.edu.cn (Zhi-Liang Zhao)
} 


\section{Introduction}

Uncertainties, including unmodeled nonlinear dynamics, external disturbances and parametric perturbations, are ubiquitous in practice. In control science and technology, it is a central issue to ensure the normal operation of control systems despite various uncertainties [1]. Motivated by this important objective, numerous control methods have been developed, such as proportional-integral-derivative control [2], adaptive control [3] and disturbance rejection methods [4-7]. Among various disturbance rejection methods, active disturbance rejection control (ADRC) has drawn lots of attention from researches due to its simplicity in practical implementation and superior performance to handle uncertainties, which has been successfully applied to flight systems [8-10], motion control systems [11, 12] and process control systems [13-15], just to name a few.

In the framework of ADRC, the extended state observer (ESO) is novelly constructed to estimate the total effect of various uncertainties, named as "total disturbance" [16], and then the control input is composed of the compensation for total disturbance and the feedback of system states. In the last two decades, the theoretical foundation of ADRC has been substantially established. For the ESO which is the vital component in ADRC, the literatures [17] investigated the convergence of ESO and the bound of estimating error. The closed-loop stability of ADRC was firstly presented in [18], where the considered uncertainties and their derivatives with respect to the time are assumed to be bounded. For the linear time-invariant uncertain systems, the literature [19] theoretically illustrated the satisfied tracking performance of ADRC despite a large scope of parametric variations. In [20], by assuming the existence of the Lyapunov functions related with uncertainties, the capability of ADRC to deal with nonlinear uncertainties was proved. By assuming that the uncertainties and their partial derivatives are bounded if the states are in a bounded set, the references $[21,22]$ rigorously studied the convergence and the transient performance of ADRC. It is remarkable that the conditions in $[21,22]$ can describe a wide class of nonlinear uncertainties in practice. By utilizing the concept of total disturbance, the literatures $[23,24]$ illuminated the capability of ADRC to handle the mismatched nonlineari- 
ties in both dynamical systems and measurement model. Besides, several successful modifications of ADRC have been made for nonlinear uncertain systems with other complicated practical factors, such as time delay $[25,26]$ and stochastic uncertainties $[27,28]$.

Up to now, the theoretical results demonstrate the effectiveness of ADRC to tackle the time-varying disturbances and the nonlinear internal uncertainties dependent on system states. In the conventional ADRC design, it is remarkable that the information of the control coefficient is required to design the compensation term for total disturbance $[7,29,30]$. In practical systems, the true value of control coefficient is usually unknown [19], which promotes the development of the ADRC based on the nominal values or approximative mathematical expression of control coefficient. For the ADRC based on the nominal information of control coefficient, the literatures [19, 21, 23] quantitatively analyzed the stability range of the uncertain control coefficient. Unfortunately, compared with the nonlinear uncertainties dependent on system states, the capability of the conventional ADRC to deal with the uncertainties of control coefficient is limited [31]. Besides, in some practical systems, it is difficult to obtain the nominal values or approximative mathematical expression of control coefficient $[13,14,25]$. Hence it is significant to design a new ADRC which is featured with strong robustness to uncertainties and does not rely on the nominal value or the approximative mathematical expression of control coefficient.

The paper studies the control problem for a class of lower-triangular nonlinear uncertain systems with mismatched uncertainties. Based on the signs of control coefficients rather than the nominal values or the mathematical expressions, a new ADRC design is proposed to handle the uncertainties caused by the mismatched nonlinearities and the unknown values of control coefficients. The design procedure of the new ADRC can be separated into three parts: 1) determining the transformation from the original states to the states of an integrators chain system; 2) designing the ESO to estimate the total disturbance and the states of the integrators chain system; 3) forcing the actual input to track the ideal input by a dynamical system. By rigorously analyzing the properties of the closed-loop system, the bounds of tracking error, estimating error and the error between the actual and ideal inputs are explicitly shown as the functions 
of control parameters. Based on the detailed expressions of error bounds, it is demonstrated that the satisfied closed-loop performance can be obtained by suitably enlarging the ESO's parameter despite a wide class of uncertainties. Moreover, by meticulously studying the relationship between the original states and integrators chain states, the paper removes the bounded assumption for integrators chain states [16, 23]. The main contributions of the paper are as follows.

(1). Based on the signs of control coefficients rather than the nominal values or the mathematical expressions, a new ADRC is proposed.

(2). By considering a large scope of nonlinear uncertainties, the satisfied transient performance of the proposed ADRC is proved.

(3). The tuning principle of the control parameters is provided. Especially, the relationship between the parameter of input dynamical system and the ESO's parameter is explicitly shown.

The rest of the paper has the following organization. In Section 2, the problem formulation is given. In Section 3, the new ADRC design based on the signs of control coefficients is proposed. In Section 4, the transient performance of the closed-loop system is presented. The simulation studies are shown in Section 5. The conclusion is presented in Section 6.

\subsection{Notations}

The following notations are used throughout the paper. $y^{(k)}(t)$ is the $k$-th order derivative of $y$ respect to the variable $t$ for $k \geq 1$ and $y^{(0)}(t) \triangleq y(t)$. The notations $|\cdot|$ and $\|\cdot\|$ are the absolute value of a scalar and the 2-norm of a vector or a matrix, respectively. The notation $\operatorname{diag}\left(a_{1}, a_{2}, \cdot, a_{m}\right)$ represents a diagonal matrix with the dimension $m \times m$, whose $i$-th diagonal element is $a_{i}$. For the real symmetric matrix $A$, the maximal eigenvalue of $A$ is denoted as $\lambda_{\max }(A)$ and the minimal eigenvalue of $A$ is 
denoted as $\lambda_{\min }(A)$. The following useful matrices are introduced.

$$
\begin{aligned}
& A=\left[\begin{array}{cccc}
0 & 1 & \cdots & 0 \\
\vdots & 0 & \ddots & \vdots \\
\vdots & \vdots & \ddots & \ddots \\
0 & 0 & \cdots & 0
\end{array}\right]_{n \times n}, B=\left[\begin{array}{c}
0 \\
\vdots \\
0 \\
1
\end{array}\right]_{n \times 1}, B_{f}=\left[\begin{array}{c}
0 \\
\vdots \\
0 \\
1
\end{array}\right]_{(n+1) \times 1}, C=\left[\begin{array}{c}
1 \\
0 \\
\vdots \\
0
\end{array}\right]_{n \times 1}, \\
& A_{e}=\left[\begin{array}{cc}
A & B \\
0 & 0
\end{array}\right]_{(n+1) \times(n+1)}, C_{e}=\left[\begin{array}{c}
C \\
0
\end{array}\right]_{(n+1) \times 1} .
\end{aligned}
$$

The function $\operatorname{sgn}(\cdot)$ represents the sign function, which satisfies

$$
\operatorname{sgn}(a)=\left\{\begin{array}{l}
1, \text { if } a>0, \\
0, \text { if } a=0, \\
-1, \text { if } a<0 .
\end{array}\right.
$$

\section{Problem formulation}

Consider the following class of lower-triangular nonlinear uncertain systems with unknown control coefficients.

$$
\left\{\begin{array}{l}
\dot{x}_{i}(t)=\theta_{i}(t) x_{i+1}(t)+\phi_{i}\left(x_{1}(t), \cdots, x_{i}(t), t\right), \quad 1 \leq i \leq n-1, \\
\dot{x}_{n}(t)=\theta_{n}(t) u(t)+\phi_{n}\left(x_{1}(t), \cdots, x_{n}(t), t\right) \\
\dot{z}(t)=g(z, x, t) \\
y(t)=x_{1}(t), \quad t \geq t_{0}
\end{array}\right.
$$

where $x(t)=\left[\begin{array}{lll}x_{1}(t) & \cdots & x_{n}(t)\end{array}\right]^{T} \in R^{n}$ and $z(t) \in R^{m}$ are the system states, $y(t) \in R$ is the measured output to be controlled, $u(t) \in R$ is the control input, $g(\cdot)$ represents the dynamics of the state $z(t), \phi_{i}(\cdot)(1 \leq i \leq n)$ represent the uncertainties in various channels which might be mismatched, and $\theta_{i}(t)(1 \leq i \leq n)$ are the control coefficients. As shown in [32], the signs of the control coefficients $\theta_{i}(t)$, i.e., $\operatorname{sgn}\left(\theta_{i}(t)\right)(1 \leq i \leq n)$, represent the control directions of the system (3). The paper considers the situation that the control directions $\operatorname{sgn}\left(\theta_{i}(t)\right)(1 \leq i \leq n)$ are known. However, both the approximative mathematical expressions and the nominal values of the control coefficients $\theta_{i}(t)$ are unknown.

Based on the control directions $\operatorname{sgn}\left(\theta_{i}(t)\right)$ rather than the nominal values or mathematical expressions of control coefficients, the control objective of the system (3) is 
to design the control input $u(t)$ such that the output $y(t)$ can track the reference signal $r(t)$ despite the multiple uncertainties $\phi_{i}(\cdot)(1 \leq i \leq n)$.

Before the detailed control design, the following assumptions for the reference signal $r(t)$, the multiple uncertainties $\phi_{i}(\cdot)(1 \leq i \leq n)$ and the dynamics of the state $z(t)$ are introduced.

Assumption 1. There exists a positive constant $M_{r}$ such that $\sup _{t \geq t_{0}}\left|r^{(i)}(t)\right| \leq M_{r}$ for $0 \leq i \leq n+1$.

Assumption 2. The functions $\phi_{i}(\cdot)$ and $\theta_{i}(\cdot)$ are $(n+1-i)$-th order differentiable with respect to their variables for $1 \leq i \leq n$. There exist positive constants $\bar{M}_{\theta, i}$ and $\underline{M}_{\theta, i}$ and continuous functions $\psi_{\phi, i}\left(x_{1}, \cdots, x_{i}\right)$ such that

$$
\begin{gathered}
\sup _{t \geq t_{0}, 0 \leq j \leq n+1-i}\left|\theta_{i}^{(j)}(t)\right| \leq \bar{M}_{\theta, i}, \quad \inf _{t \geq t_{0}}\left|\theta_{i}(t)\right| \geq \underline{M}_{\theta, i}>0, \\
\sup _{t \geq t_{0}}\left|\frac{\partial^{\Sigma_{k=1}^{i+1} j_{k}} \phi_{i}\left(x_{1}, \cdots, x_{i}, t\right)}{\partial x_{1}^{j_{1}} \cdots \partial x_{i}^{j_{i}} \partial t^{j_{i+1}}}\right| \leq \psi_{\phi, i}\left(x_{1}, \cdots, x_{i}\right),
\end{gathered}
$$

for $\Sigma_{k=1}^{i+1} j_{k} \leq n+i-1,1 \leq i \leq n$ and $j_{p} \geq 0(1 \leq p \leq i+1)$.

Assumption 3. There exists a radially unbounded positive-definite function $V_{z}(z)$ such that

$$
\dot{V}_{z}(z(t))=\frac{d V_{z}}{d z} g(z, x, t) \leq 0, \quad \forall V_{z}(z) \geq r_{z}(\|x\|), \quad t \geq t_{0},
$$

where $r_{z}(\cdot)$ is a nonnegative continuous increasing function.

Assumption 1 implies that the reference signal and its derivatives are bounded, which is rational in practice [29]. Assumption 2 assumes that the control coefficients and their derivatives are bounded. Moreover, (4) implies that the control coefficients are not equal to zero for $t \geq t_{0}$ which ensures the controllability of the system (3). From (5), the uncertainties $\phi_{i}(\cdot)$ are bounded by some continuous functions $\psi_{\phi, i}(\cdot)$ dependent on the system states, which allows a large scope of internal uncertainties and external disturbances in practice. By regarding $x(t)$ as the input of the $z$-subsystem, Assumption 3 implies that the $z$-subsystem is uniformly input-state-stable [21]. For the system (3) being a linear time invariant system, Assumption 3 is equivalent to that the system (3) is a minimum phase plant. 
In the next section, an ADRC design based on the control directions $\operatorname{sgn}\left(\theta_{i}(t)\right)$ is proposed to achieve the tracking objective despite multiple uncertainties.

Remark 1. As shown in the conventional ADRC design [7, 29], the control coefficient

plays an important role in the design of ESO and feedback term. However, it is sometimes hard to acquire the nominal values or the mathematical expressions of control coefficients in practical systems [13, 14]. Moreover, due to the physical mechanism, the control directions, i.e., the signs of the control coefficients, can be determined. Hence the paper aims to develop a new ADRC design based on the control directions rather than the nominal values or the approximative mathematical expressions of control coefficients.

\section{ADRC design based on control directions}

In this section, a new ADRC design based on control directions is proposed. The rest of this section consists of the following three parts.

1. The equivalent integrators chain form for the system (3) is investigated, which shows the essential relationship from the input to the output. Moreover, the output regulation task is transmitted to the state tracking task.

2. Based on the integrators chain system, an ESO is presented to estimate the derivatives of the output and the unknown term named as "total disturbance".

3. Via the estimations from ESO, the control input is generated by a dynamical system, which forces the input to track the ideal input signal.

\subsection{Analysis for the equivalent integrators chain form}

In this subsection, the equivalent integrators chain form for the system (3) is investigated. 
Denote the new state vector $\tilde{x}(t)=\left[\begin{array}{llll}\tilde{x}_{1}(t) & \cdots & \tilde{x}_{n}(t)\end{array}\right]^{T}$ as follows.

$$
\left\{\begin{aligned}
\tilde{x}_{1}(t)= & x_{1}(t), \\
\tilde{x}_{i}(t)= & \dot{\tilde{x}}_{i-1}(t) \\
= & \left(\prod_{j=1}^{i-1} \theta_{j}(t)\right) x_{i}(t)+\sum_{j=1}^{i-2} \frac{d^{j-1}}{d t^{j-1}}\left(\frac{d\left(\prod_{k=1}^{i-j-1} \theta_{k}(t)\right)}{d t} x_{i-j}(t)\right) \\
& +\sum_{j=1}^{i-1} \frac{d^{j-1}}{d t^{j-1}}\left(\left(\prod_{k=0}^{i-j-1} \theta_{k}(t)\right) \phi_{i-j}\left(x_{1}, \cdots, x_{i-j}, t\right)\right), 2 \leq i \leq n,
\end{aligned}\right.
$$

where $\theta_{0}(t)=1$ for $t \geq t_{0}$.

The following lemma illustrates the relationship between the state vectors $x$ and $\tilde{x}$.

Lemma 1. Consider the transformation (7) with Assumption 2. Then, there exist two continuous mappings satisfying

$$
\gamma(x, t) \triangleq\left[\begin{array}{c}
\gamma_{1}(x, t) \\
\vdots \\
\gamma_{n+1}(x, t)
\end{array}\right]=\left[\begin{array}{c}
\tilde{x}_{1} \\
\vdots \\
\tilde{x}_{n} \\
t
\end{array}\right], \quad \varphi(\tilde{x}, t) \triangleq\left[\begin{array}{c}
\varphi_{1}(\tilde{x}, t) \\
\vdots \\
\varphi_{n+1}(\tilde{x}, t)
\end{array}\right]=\left[\begin{array}{c}
x_{1} \\
\vdots \\
x_{n} \\
t
\end{array}\right]
$$

and

$$
\begin{gathered}
\sup _{t \geq t_{0},\|x\| \leq \rho_{x}}\left\{\left|\gamma_{i}(x, t)\right|,\left\|\frac{\partial \gamma_{i}(x, t)}{\partial x}\right\|,\left|\frac{\partial \gamma_{i}(x, t)}{\partial t}\right|\right\} \leq \psi_{\gamma}\left(\rho_{x}\right), \quad \forall \rho_{x} \geq 0, \forall 1 \leq i \leq n, \\
\sup _{t \geq t_{0},\|\tilde{x}\| \leq \tilde{\rho}_{x}}\left\{\left|\varphi_{i}(\tilde{x}, t)\right|,\left\|\frac{\partial \varphi_{i}(\tilde{x}, t)}{\partial \tilde{x}}\right\|,\left|\frac{\partial \varphi_{i}(\tilde{x}, t)}{\partial t}\right|\right\} \leq \psi_{\varphi}\left(\tilde{\rho}_{x}\right), \quad \forall \tilde{\rho}_{x} \geq 0, \forall 1 \leq i \leq n,
\end{gathered}
$$

where $\psi_{\gamma}(\cdot)$ and $\psi_{\varphi}(\cdot)$ are continuous increasing functions dependent on $\bar{M}_{\theta, i}, \underline{M}_{\theta, i}$ and $\psi_{\phi, i}$ for $1 \leq i \leq n$.

The proof of Lemma 1 is given in Appendix.

Remark 2. In the existing studies [16, 23], the bounds of the mappings $\gamma_{i}(\cdot)$ and $\varphi_{i}(\cdot)$ are provided as an additional assumption. In this paper, by rigorously analyzing the detailed expressions of the mappings $\gamma_{i}(\cdot)$ and $\varphi_{i}(\cdot)$, we prove that $\gamma_{i}(\cdot)$ and $\varphi_{i}(\cdot)$ and their partial derivatives are bounded, i.e., (9)-(10). Hence the assumptions for the bounds of $\gamma_{i}(\cdot)$ and $\varphi_{i}(\cdot)$ are removed in the paper. 
Based on the transformation (7) and Lemma 1, the system (3) can be rewritten as the following integrators chain system.

$$
\left\{\begin{array}{l}
\dot{\tilde{x}}_{i}(t)=\tilde{x}_{i+1}(t), \quad 1 \leq i \leq n-1 \\
\dot{\tilde{x}}_{n}(t)=b(t) u(t)+f(\tilde{x}(t), t) \\
\dot{z}(t)=g\left(z, \varphi_{1}(\tilde{x}, t), \cdots, \varphi_{n}(\tilde{x}, t), t\right) \\
y(t)=\tilde{x}_{1}(t), \quad t \geq t_{0}
\end{array}\right.
$$

with the initial condition $\tilde{x}\left(t_{0}\right)=\left[\gamma_{1}\left(x\left(t_{0}\right), t_{0}\right) \cdots \gamma_{n}\left(x\left(t_{0}\right), t_{0}\right)\right]^{T}$. The control coefficient $b(t)$ and the uncertainty $f(\tilde{x}(t), t)$ have the following form.

$$
\left\{\begin{array}{l}
b(t)=\Pi_{i=1}^{n} \theta_{i}(t), \\
f(\tilde{x}, t)=\sum_{j=1}^{n-1} \frac{d^{j-1}}{d t^{j-1}}\left(\frac{d\left(\Pi_{k=1}^{n-j} \theta_{k}(t)\right)}{d t} \varphi_{n+1-j}(\tilde{x}, t)\right) \\
\quad+\Sigma_{j=1}^{n} \frac{d^{j-1}}{d t^{j-1}}\left(\left(\Pi_{k=0}^{n-j} \theta_{k}(t)\right) \tilde{\phi}_{n+1-j}(\tilde{x}, t)\right),
\end{array}\right.
$$

where $\tilde{\phi}_{i}(\tilde{x}, t)=\phi_{i}\left(\varphi_{1}(\tilde{x}, t), \cdots, \varphi_{i}(\tilde{x}, t), t\right)$ for $1 \leq i \leq n$.

If the nominal value of the control coefficient $b(t)$, denoted as $\bar{b}(t)$, can be obtained, the conventional ADRC design can be applied to the system (11) by regarding $f(\tilde{x}, t)+$ $(b(t)-\bar{b}(t)) u(t)$ as the total disturbance [23, 29]. However, only the sign of the control coefficient $b(t)$ is known in the paper, rather than the nominal value or the mathematical expression.

$$
\operatorname{sgn}(b(t))=\Pi_{i=1}^{n} \operatorname{sgn}\left(\theta_{i}(t)\right) .
$$

Next, based on the integrators chain form (11) and the sign of control coefficient $b(t)$, an ADRC design will be proposed.

\subsection{ESO design}

Since the nominal value of the control coefficient $b(t)$ is unknown, the total disturbance of the system (11) is denoted as

$$
f_{t}(\tilde{x}, u, t) \triangleq b(t) u(t)+f(\tilde{x}, t) .
$$

Then the following ESO is presented to estimate $\tilde{x}$ and $f_{t}$.

$$
\left[\begin{array}{c}
\dot{\tilde{x}}(t) \\
\dot{\hat{f}}_{t}(t)
\end{array}\right]=A_{e}\left[\begin{array}{c}
\hat{\tilde{x}}(t) \\
\hat{f}_{t}(t)
\end{array}\right]+L_{e}\left(y(t)-C_{e}^{T}\left[\begin{array}{c}
\hat{\tilde{x}}(t) \\
\hat{f}_{t}(t)
\end{array}\right]\right),
$$


where $\hat{\tilde{x}}(t)=\left[\begin{array}{lll}\hat{\tilde{x}}_{1}(t) & \cdots & \hat{\tilde{x}}_{n}(t)\end{array}\right]^{T} \in R^{n}$ is the estimation for the system state vector $\tilde{x}(t)$ and $\hat{f}_{t}(t) \in R$ is the estimation for the total disturbance $f_{t}(\tilde{x}, u, t)$. In addition, the constant vector $L_{e} \in R^{(n+1) \times 1}$ is the tunable parameter vector of ESO such that the matrix $A_{L} \triangleq A_{e}-L_{e} C_{e}^{T}$ is Hurwitz. Owing to [33], the following concise tuning method of $L_{e}$ is presented.

$$
L_{e}=\left[\begin{array}{llll}
\varsigma_{1} \omega_{o} & \varsigma_{2} \omega_{o}^{2} & \cdots & \varsigma_{n+1} \omega_{o}^{n+1}
\end{array}\right]^{T}, \quad \varsigma_{i}=\frac{(n+1) !}{(n+1-i) ! i !}, \quad \omega_{o} \geq 0,
$$

which ensures that all the eigenvalues of $A_{L}$ are set at $-\omega_{o}$.

Next, a dynamical design of ADRC input based on the estimations from ESO will be presented.

\subsection{Dynamical input design}

Firstly, the ideal control input is introduced. For the system (11), the desired closedloop system satisfies the following form.

$$
\left\{\begin{array}{l}
\dot{\tilde{x}}_{i}^{*}(t)=\tilde{x}_{i+1}^{*}(t), \quad 1 \leq i \leq n-1, \\
\dot{\tilde{x}}_{n}^{*}(t)=-K^{T}\left(\tilde{x}^{*}(t)-\bar{r}(t)\right)+r^{(n)}(t), \\
\dot{z}^{*}(t)=g\left(z^{*}, \varphi_{1}\left(\tilde{x}^{*}, t\right), \cdots, \varphi_{n}\left(\tilde{x}^{*}, t\right), t\right), \\
y^{*}(t)=\tilde{x}_{1}^{*}(t), \quad t \geq t_{0}, \quad \tilde{x}^{*}\left(t_{0}\right)=\tilde{x}\left(t_{0}\right),
\end{array}\right.
$$

where $x^{*}(t)=\left[x_{1}^{*}(t) \cdots x_{n}^{*}(t)\right] \in R^{n}$ and $z^{*}(t) \in R^{m}$ are the states of the desired system, $y^{*}(t) \in R$ is the desired output, $\bar{r}(t)=\left[r(t) \cdots r^{(n-1)}(t)\right]^{T} \in R^{n}$ and the constant vector ${ }_{155} K \in R^{n \times 1}$ is feedback gain vector satisfying that the matrix $A_{K} \triangleq A-B K^{T}$ is Hurwitz.

Remark 3. For the desired system (17), the output $y^{*}(t)$ can exponentially converge to the reference signal $r(t)$ with the desired convergence rate by tuning the feedback gain vector $K$. Moreover, the system states $\left(\tilde{x}(t), z^{*}(t)\right)$ are bounded for $t \geq t_{0}$.

By comparing the integrators chain system (11) with the desired system (17), the ideal control input can be obtained as follows.

$$
u^{*}(t)=\frac{-f(\tilde{x}, t)-K^{T}(\tilde{x}(t)-\bar{r}(t))+r^{(n)}(t)}{b(t)} .
$$


Inspired by the approximative dynamic inversion method in [34, 35], the actual control input is generated by the following dynamics.

$$
\dot{u}(t)=-\operatorname{sgn}(b(t)) \kappa\left(\omega_{o}\right)\left(\hat{f}_{t}(t)+K^{T}(\hat{\tilde{x}}(t)-\bar{r}(t))-r^{(n)}(t)\right),
$$

where $\kappa\left(\omega_{o}\right)>0$ is a function to be designed.

The parameter $\kappa\left(\omega_{o}\right)$ should be carefully selected to ensure that the dynamics (19) is "slower" than the dynamics of the ESO (15) [35]. However, the explicit tuning law of $\kappa\left(\omega_{o}\right)$ has not been provided in the existing studies. To make the proposed method more friendly to practitioners, the tuning law of $\kappa\left(\omega_{o}\right)$ is investigated and is explicitly presented in the following assumption.

Assumption 4. The increasing function $\kappa\left(\omega_{o}\right)>0$ for $\omega_{o}>0$ and

$$
\lim _{\omega_{o} \rightarrow \infty} \frac{\ln \omega_{o}}{\sqrt{\kappa\left(\omega_{o}\right)}}=0, \quad \lim _{\omega_{o} \rightarrow \infty} \frac{\kappa\left(\omega_{o}\right)}{\omega_{o}}=0 .
$$

Assumption 4 describes the growth rate of the increasing function $\kappa\left(\omega_{o}\right)$ which grows faster than the $\log$ function $f_{1}\left(\omega_{o}\right)=\left(\ln \omega_{o}\right)^{2}$ and slower than the linear function $f_{2}\left(\omega_{o}\right)=\omega_{o}$. It is remarkable that the function $\kappa\left(\omega_{o}\right)=\omega_{o}^{k}$ with $0<k<1$ can satisfy Assumption 4.

Remark 4. The ideology of approximative dynamic inversion method is to design the dynamics of $u(t)$ which satisfies the following equation.

$$
\dot{u}(t)=-a(t)\left(u(t)-u^{*}(t)\right)
$$

where $a(t)>0$ is a function to be designed. By substituting (18) into (21), we have

$$
\begin{aligned}
\dot{u}(t) & =-\frac{a(t)}{b(t)}\left(b(t) u(t)+f(\tilde{x}, t)+K^{T}(\tilde{x}-\bar{r}(t))-r^{(n)}(t)\right) \\
& =-\frac{a(t)}{b(t)}\left(f_{t}(\tilde{x}, u, t)+K^{T}(\tilde{x}-\bar{r}(t))-r^{(n)}(t)\right) .
\end{aligned}
$$

By designing $a(t)=\kappa\left(\omega_{o}\right)|b(t)|$ and substituting the estimations $\left(\hat{f}_{t}, \hat{\tilde{x}}\right)$ into (22), then 


\section{Performance analysis of the closed-loop system}

The performance of the closed-loop system based on the ADRC design (15) and (19) will be investigated in this section.

The following theorem shows the satisfactory transient performance of the closedloop system based on the proposed ADRC despite a wide class of uncertainties.

Theorem 1. Consider the system (3) with Assumptions 1-4. Let $u(t)=0$ for $t \in\left[t_{0}, t_{u}\right)$ where

$$
\left\{\begin{array}{l}
t_{u}=t_{0}+2 n c_{\varsigma 2} \frac{\max \left\{\ln \left(\omega_{o} \rho_{0}\right), 0\right\}}{\sqrt{\kappa\left(\omega_{o}\right)}}, \quad \rho_{0}=\max _{2 \leq i \leq n}\left|\tilde{x}_{i}\left(t_{0}\right)-\hat{\tilde{x}}_{i}\left(t_{0}\right)\right|^{\frac{1}{n}}, \\
c_{\varsigma 2}=\lambda_{\max }\left(P_{\zeta}\right), \quad A_{\varsigma}^{T} P_{\varsigma}+P_{\varsigma} A_{\varsigma}=-I, A_{\varsigma}=\left[\begin{array}{ccccc}
-\varsigma_{1} & 1 & 0 & \cdots & 0 \\
\vdots & 0 & \ddots & \ddots & \vdots \\
\vdots & \vdots & \ddots & \ddots & 0 \\
-\varsigma_{n} & 0 & \ldots & 0 & 0 \\
-\varsigma_{n+1} & 0 & \ldots & \ldots & 0
\end{array}\right] .
\end{array}\right.
$$

For $t \geq t_{u}, u(t)$ is designed according to (15) and (19). Then there exist positive constants $\eta_{i}^{*}(1 \leq i \leq 5)$ and $\omega^{*}$ dependent on $\left(x\left(t_{0}\right), \hat{\tilde{x}}\left(t_{0}\right), \hat{f}_{t}\left(t_{0}\right), M_{r}, \underline{M}_{\theta, i}, \bar{M}_{\theta, i}, \psi_{\phi i}, K\right)$ such that

$$
\begin{aligned}
& \sup _{t \geq t_{0}}\left|y(t)-y^{*}(t)\right| \leq \eta_{1}^{*} \max \left\{\frac{\ln \omega_{o}}{\sqrt{\kappa\left(\omega_{o}\right)}}, \frac{\kappa\left(\omega_{o}\right)}{\omega_{o}}, \frac{1}{\kappa\left(\omega_{o}\right)}\right\}, \\
& \left\|\left[\begin{array}{c}
\tilde{x}(t)-\hat{\tilde{x}}(t) \\
f_{t}(\tilde{x}, u, t)-\hat{f}_{t}(t)
\end{array}\right]\right\| \leq \eta_{2}^{*}\left(\frac{\kappa\left(\omega_{o}\right)}{\omega_{o}}+e^{-\eta_{3}^{*} \omega_{o}\left(t-t_{u}\right)}\right), \forall t \geq t_{u}, \\
& \left|u(t)-u^{*}(t)\right| \leq \eta_{4}^{*}\left(\frac{\kappa\left(\omega_{o}\right)}{\omega_{o}}+\frac{1}{\kappa\left(\omega_{o}\right)}+e^{-\eta_{5}^{*} \kappa\left(\omega_{o}\right)\left(t-t_{u}\right)}\right), \forall t \geq t_{u},
\end{aligned}
$$

for any $\omega_{o} \geq \omega^{*}$

Theorem 1 demonstrates that the tracking error between the actual and ideal outputs, the estimating error of the ESO (15) and the error between the actual and ideal inputs are bounded. Furthermore, (24)-(26) explicitly show the bounds of the tracking error, estimating error and the error between the actual and ideal inputs. More importantly, as shown in (24), the tracking error between the actual and ideal outputs can be sufficiently small for $t \geq t_{0}$ by tuning $\omega_{o}$ to be suitably large, which ensures the satisfied transient performance despite various uncertainties. In addition, (25)-(26) implies that 
both the estimating error and the error between the actual and ideal inputs can converge into a neighbourhood with a small boundary by suitably enlarging $\omega_{o}$, which illustrates the tuning law of the parameter $\omega_{o}$.

Remark 5. It is remarkable that the satisfied closed-loop performance despite a large scope of uncertainties can be achieved by tuning one scale parameter, which is friendly to practical practitioner.

Remark 6. The design (23) is a common way to avoid the peaking phenomenon of ESO [21]. Moreover, since plenty of practical systems satisfy the initial condition that $\tilde{x}=\left[\begin{array}{lll}0 & \cdots & 0\end{array}\right]^{T}$, it can be obtained by designing $\hat{\tilde{x}}=\left[\begin{array}{lll}0 & \cdots & 0\end{array}\right]^{T}$ that $t_{u}=t_{0}$. In addition, for the systems satisfying $\rho \geq \frac{1}{\omega_{o}}$, it can be deduced from (23) and Assumption 4 that $t_{u}$ can be closed to $t_{0}$ by suitably enlarging $\omega_{o}$.

To simplify the proof of Theorem 1, Lemmas 2-3 are introduced. Lemma 2 describes the bounds of the uncertainty $f(\tilde{x}, t)$, the control coefficient $b(t)$ and their derivatives. Lemma 3 provides the closed-loop form and the bounds of the uncertain terms in the closed-loop system. The proofs of Lemmas 2-3 are given in Appendix.

Lemma 2. Let Assumption 2 hold. For any given positive constant $\tilde{\rho}_{x}$, the functions $b(t)$ and $f(\tilde{x}, t)$ in (12) satisfy the following equations:

$$
\begin{gathered}
\sup _{t \geq t_{0}}\left\{\left|(b(t))^{-1}\right|,|b(t)|,|\dot{b}(t)|\right\} \leq \psi_{b}, \\
\sup _{t \geq t_{0},\|\tilde{x}\| \leq \tilde{\rho}_{x}}\left\{|f(\tilde{x}, t)|,\left\|\frac{\partial f(\tilde{x}, t)}{\partial \tilde{x}}\right\|,\left|\frac{\partial f(\tilde{x}, t)}{\partial t}\right|\right\} \leq \psi_{f}\left(\tilde{\rho}_{x}\right),
\end{gathered}
$$

where the positive constant $\psi_{b}$ and the non-decreasing function $\psi_{f}(\cdot)$ are dependent on $\bar{M}_{\theta, i}, \underline{M}_{\theta, i}$ and $\psi_{\phi, i}$ for $1 \leq i \leq n$.

Denote the tracking error vector, estimating error vector and the error between the actual and ideal control inputs as follows.

$$
e(t)=\tilde{x}(t)-\tilde{x}^{*}(t), \quad \zeta(t)=T_{1}^{-1}\left[\begin{array}{c}
\tilde{x}(t)-\hat{\tilde{x}}(t) \\
f_{t}(\tilde{x}, u, t)-\hat{f}_{t}(t)
\end{array}\right], \quad \delta_{u}(t)=u(t)-u^{*}(t),
$$


where $T_{1}=\operatorname{diag}\left(\omega_{o}^{-n}, \cdots, \omega_{o}^{-1}, 1\right)$. Then the following lemma presents the closed-loop system and further analyzes the properties of the uncertain terms in the closed-loop system.

Lemma 3. Let Assumptions 1-3 hold. Design $u(t)=0$ for $t \in\left[t_{0}, t_{u}\right)$, and design $u(t)$ by (15) and (19) for $t \in\left[t_{u}, \infty\right)$. Then the closed-loop system is shown as follow.

$$
\begin{aligned}
& \left\{\begin{array}{l}
\dot{e}(t)=A e(t)+B \Delta_{e 0}(e, t), \\
\dot{z}(t)=g\left(z, \varphi_{1}\left(\tilde{x}^{*}+e, t\right), \cdots, \varphi_{n}\left(\tilde{x}^{*}+e, t\right), t\right), \quad t \in\left[t_{0}, t_{u}\right), \\
\dot{\zeta}(t)=\omega_{o} A_{\zeta} \zeta(t)+B_{f} \Delta_{\zeta 0}(e, t), \\
\dot{\delta}_{u}(t)=-|b(t)| \kappa \delta_{u}(t)+\Delta_{\delta_{u} 0}\left(e, \zeta, \omega_{o}, \kappa, t\right),
\end{array}\right. \\
& \left\{\begin{array}{l}
\dot{e}(t)=A_{K} e(t)+B \Delta_{e 1}\left(\delta_{u}, t\right), \\
\dot{z}(t)=g\left(z, \varphi_{1}\left(\tilde{x}^{*}+e, t\right), \cdots, \varphi_{n}\left(\tilde{x}^{*}+e, t\right), t\right), \\
\dot{\zeta}(t)=\omega_{o} A_{\zeta} \zeta(t)+B_{f} \Delta_{\zeta 1}\left(e, \zeta, \delta_{u}, \omega_{o}, \kappa, t\right), \\
\dot{\delta}_{u}(t)=-|b(t)| \kappa \delta_{u}(t)+\Delta_{\delta_{u} 1}\left(e, \zeta, \delta_{u}, \omega_{o}, \kappa, t\right),
\end{array} \quad t \in\left[t_{u}, \infty\right) .\right.
\end{aligned}
$$

Moreover, the uncertain terms $\left(\Delta_{e 0}, \Delta_{\zeta 0}, \Delta_{\delta_{u} 0}, \Delta_{e 1}, \Delta_{\zeta 1}, \Delta_{\delta_{u} 1}\right)$ have the following bounds.

$$
\left\{\begin{array}{l}
\left|\Delta_{e 0}\right| \leq \pi_{e 0}\left(\rho_{e}\right), \quad\left|\Delta_{e 1}\right| \leq \psi_{b}\left|\delta_{u}\right|, \\
\left|\Delta_{\zeta 0}\right| \leq \pi_{\zeta 0}\left(\rho_{e}\right), \quad\left|\Delta_{\zeta 1}\right| \leq \pi_{\zeta 1}\left(\rho_{e}\right)+\pi_{\omega}\left(\omega_{o}^{*}\right) \kappa\|\zeta\|+(\kappa+1) \pi_{\delta_{u}}\left(\rho_{e}, \rho_{u}\right), \\
\left|\Delta_{\delta_{u} 0}\right| \leq \pi_{\delta_{u} 0}\left(\rho_{e}\right)+\pi_{\omega}\left(\omega_{o}^{*}\right) \kappa\|\zeta\|, \quad\left|\Delta_{\delta_{u} 1}\right| \leq \pi_{\delta_{u} 1}\left(\rho_{e}, \rho_{u}\right)+\pi_{\omega}\left(\omega_{o}^{*}\right) \kappa\|\zeta\|,
\end{array}\right.
$$

for $e \in\left\{e \mid\|e\| \leq \rho_{e}\right\}, \zeta \in\left\{\zeta \mid\|\zeta\| \leq \rho_{\zeta}\right\}, \delta_{u} \in\left\{\delta_{u}|| \delta_{u} \mid \leq \rho_{u}\right\}$ and $\omega_{o} \in\left\{\omega_{o} \mid \omega_{o} \geq \omega_{o}^{*}\right\}$ with any given positives $\rho_{e}, \rho_{\zeta}, \rho_{u}$ and $\omega_{o}^{*}$. The functions $\pi_{e 0}(\cdot), \pi_{\zeta 0}(\cdot), \pi_{\zeta 1}(\cdot), \pi_{\delta_{u}}(\cdot)$, $\pi_{\delta_{u} 0}(\cdot)$ and $\pi_{\delta_{u} 1}(\cdot)$ are non-decreasing and are dependent on $K, M_{r}, \bar{M}_{\theta, i}, \underline{M}_{\theta, i}$ and $\psi_{\phi, i}$ for $1 \leq i \leq n$. The function $\pi_{\omega}(\cdot)$ is non-increasing and is dependent on $\bar{M}_{\theta, i}, \underline{M}_{\theta, i}$ and $K$.

Based on Lemmas 2-3, the proof of Theorem 1 is presented as follows.

Proof 1 (Proof of Theorem 1). With the mapping from $(x, t)$ to $(\tilde{x}, t)$ presented in Lemma 1 and the discussions in Section 3.1, the plant (3) can be rewritten as the integrators chain system (11). Owing to Lemmas 2-3, the closed-loop system is formulated as (30)-(31). 
Since the matrices $A_{K}$ and $A_{\zeta}$ are Hurwitz, there exist positive definite matrices $P_{K}$ and $P_{\varsigma}$ such that $A_{K}^{T} P_{K}+P_{K} A_{K}=-I$ and $A_{\varsigma}^{T} P_{\varsigma}+P_{\varsigma} A_{\varsigma}=-I$. Then the following Lyapunov functions are introduced.

$$
V_{K}(t)=e^{T}(t) P_{K} e(t), \quad V_{\zeta}(t)=\zeta^{T}(t) P_{\zeta} \zeta(t), \quad V_{u}(t)=\frac{\delta_{u}^{2}(t)}{2} .
$$

Let $c_{k 1}$ and $c_{k 2}$ be the minimal and maximal eigenvalues of $P_{K}$, and denote $c_{\varsigma}$ and $c_{\zeta 2}$ as the minimal and maximal eigenvalues of $P_{\varsigma}$. Then the following inequalities hold.

$$
c_{k 1}\|e(t)\|^{2} \leq V_{K}(t) \leq c_{k 2}\|e(t)\|^{2}, \quad c_{\zeta 1}\|\zeta(t)\|^{2} \leq V_{\varsigma}(t) \leq c_{\zeta 2}\|\zeta(t)\|^{2} .
$$

Next, we analyze the properties of the closed-loop system for $t \in\left[t_{0}, t_{u}\right)$ and $t \in\left[t_{u}, \infty\right)$.

Part 1: The analysis for the closed-loop system for $t \in\left[t_{0}, t_{u}\right)$.

Owing to (11) and (17), we get the initial condition $e\left(t_{0}\right)=0$. The dynamics (30) implies the continuity of $e(t)$. Hence for a sufficiently small positive $\Delta t$ satisfying $t_{0}+$ $\Delta t \leq t_{u}$, there exists a positive constant $\eta_{e 1}$ such that

$$
\|e(t)\| \leq \eta_{e 1}, \quad \forall t \in\left[t_{0}, t_{0}+\Delta t\right] .
$$

According to Assumption 4, it can be verified from that $\lim _{\omega_{o} \rightarrow \infty} \frac{\ln \omega_{o}}{\sqrt{\kappa\left(\omega_{o}\right)}}=0$. In addition, it can be verified from (23) that $\lim _{\omega_{o} \rightarrow \infty} t_{u}=t_{0}$. Hence there exists a positive constant $\omega_{1}$ such that $t_{u}-t_{0} \leq \Delta t$ for $\omega_{o} \geq \omega_{1}$. Combined with the statement (35), it can be concluded that $\|e(t)\| \leq \eta_{e 1}$ for $t \in\left[t_{0}, t_{u}\right)$ and $\omega_{o} \geq \omega_{1}$.

Due to Lemma 1 , the bound of $x(t)$ for $t \in\left[t_{0}, t_{u}\right)$ satisfies that $\sup _{t_{0} \leq t \leq t_{u}}\|x(t)\| \leq$ $n \psi_{\varphi}\left(\eta_{e 1}+M_{x^{*}}\right)$. Combined with Assumption 3, the following equation is satisfied.

$$
\dot{V}_{z}(z(t)) \leq 0, \quad \forall V_{z}(z) \geq r_{z}\left(n \psi_{\varphi}\left(\eta_{e 1}+M_{x^{*}}\right)\right), t_{0} \leq t \leq t_{u},
$$

which further implies that

$$
\sup _{t_{0} \leq t \leq t_{u}} V_{z}(z(t)) \leq \eta_{V_{z} 1} \triangleq r_{z}\left(n \psi_{\varphi}\left(\eta_{e 1}+M_{x^{*}}\right)\right) .
$$

Based on the bound of $e(t)$ for $t \in\left[t_{0}, t_{u}\right)$, the dynamics (30) implies that

$$
\begin{aligned}
\sup _{t_{0} \leq t \leq t_{u}}\|e(t)\| & \leq\left(t_{u}-t_{0}\right) \cdot \sup _{t_{0} \leq t \leq t_{u}}\left\{\|A\|\|e(t)\|+\|B\|\left|\Delta_{e 0}(e, t)\right|\right\} \\
& \leq 4 n c_{\varsigma 2}\left(\|A\| \eta_{e 1}+\pi_{e 0}\left(\eta_{e 1}\right)\right) \frac{\ln \omega_{o}}{\sqrt{\kappa}} .
\end{aligned}
$$


Due to (30) and (32), the following dynamics of $\sqrt{V_{\varsigma}(t)}$ and $\sqrt{V_{u}(t)}$ for $t \in\left[t_{0}, t_{u}\right)$ hold for $\sqrt{V_{\varsigma}(t)} \neq 0$ and $\sqrt{V_{u}(t)} \neq 0$.

$$
\left\{\begin{aligned}
\frac{d \sqrt{V_{\zeta}(t)}}{d t} & =\frac{-\omega_{o}\|\zeta\|^{2}+2 \zeta^{T} P_{\zeta} B_{f} \Delta_{\zeta 0}}{2 \sqrt{V_{\zeta}}} \leq-\frac{\omega_{o}}{2 c_{\zeta 2}} \sqrt{V_{\zeta}(t)}+\frac{\left\|P_{\zeta}\right\| \pi_{\zeta 0}\left(\eta_{e 1}\right)}{\sqrt{c_{\zeta 1}}} \\
\frac{d \sqrt{V_{u}(t)}}{d t} & =\frac{-|b| \kappa\left|\delta_{u}\right|^{2}+\delta_{u} \Delta_{\delta_{u} 0}}{2 \sqrt{V_{u}}} \\
& \leq-\psi_{b}^{-1} \kappa \sqrt{V_{u}(t)}+\frac{\sqrt{2} \pi_{\delta_{u} 0}\left(\eta_{e 1}+\sqrt{2} \pi_{\omega}\left(\omega_{1}\right) \kappa\|\zeta(t)\|\right.}{2}
\end{aligned}\right.
$$

for $\omega_{o} \geq \omega_{1}$. With the help of Gronwall lemma, it can be deduced from (39) that $\sqrt{V_{\varsigma}(t)}$ has the following bound.

$$
\sqrt{V_{\varsigma}(t)} \leq \frac{2 c_{\varsigma 2}\left\|P_{\zeta}\right\| \pi_{\zeta 0}\left(\eta_{e 1}\right)}{\sqrt{c_{\zeta 1}} \omega_{o}}+\sqrt{V_{\varsigma}\left(t_{0}\right)} e^{-\frac{\omega_{o}\left(t-t_{0}\right)}{2 c_{\zeta 2}}}, \quad t \in\left[t_{0}, t_{u}\right] .
$$

Based on Gronwall lemma and (39)-(40), the bound of $\sqrt{V_{u}(t)}$ is obtained as follows.

$$
\begin{aligned}
\sqrt{V_{u}(t)} \leq & \frac{\sqrt{2} \pi_{\delta_{u} 0}\left(\eta_{e 1}\right)}{2 \psi_{b}^{-1} \kappa}+\sqrt{V_{u}\left(t_{0}\right)} e^{-\psi_{b}^{-1} \kappa\left(t-t_{0}\right)}+\int_{t_{0}}^{t} e^{-\psi_{b}^{-1} \kappa(t-s)} \sqrt{2} \pi_{\omega} \kappa\|\zeta(s)\| d s \\
\leq & \frac{\sqrt{2} \pi_{\delta_{u} 0}\left(\eta_{e 1}\right)}{2 \psi_{b}^{-1} \kappa}+\frac{\sqrt{2} \pi_{\omega} c_{\zeta 2}\left\|P_{\zeta}\right\| \pi_{\zeta 0}\left(\eta_{e 1}\right)}{c_{\zeta 1} \psi_{b}^{-1} \omega_{o}}+\sqrt{V_{u}\left(t_{0}\right)} e^{-\psi_{b}^{-1} \kappa\left(t-t_{0}\right)} \\
& +\frac{\sqrt{2} \pi_{\omega} \kappa \sqrt{V_{\zeta}\left(t_{0}\right)}}{\sqrt{c_{\zeta 1}}\left(\frac{\omega_{o}}{2 c_{\zeta 2}}-\psi_{b}^{-1} \kappa\right)} e^{-\psi_{b}^{-1} \kappa\left(t-t_{0}\right)}\left(1-e^{-\left(\frac{\omega_{o}}{2 c_{\zeta 2}}-\psi_{b}^{-1} \kappa\right)\left(t-t_{0}\right)}\right) .
\end{aligned}
$$

Next the bounds of $\sqrt{V_{\mathcal{\zeta}}\left(t_{u}\right)}$ and $\sqrt{V_{u}\left(t_{u}\right)}$ will be analyzed.

Consider the initial condition satisfying $\rho_{0}>\frac{1}{\omega_{o}}$. The initial value of $\sqrt{V_{\varsigma}}$ satisfies that $\sqrt{V_{\varsigma}\left(t_{0}\right)} \leq n \sqrt{c_{c_{2}}} \omega_{o}^{n-1} \rho_{0}^{n-1}$. Owing to the definition of $t_{u}$ (23), there is

$$
\left\{\begin{array}{l}
\sqrt{V_{\zeta}\left(t_{0}\right)} e^{-\frac{\omega_{o}\left(t_{u}-t_{0}\right)}{2 c_{\zeta 2}}}=\sqrt{V_{\varsigma}\left(t_{0}\right)} e^{-\frac{n \omega_{o}}{\sqrt{\kappa}} \ln \left(\omega_{o} \rho_{0}\right)} \leq \frac{n \sqrt{c_{\zeta 2}}}{\omega_{o} \rho_{0}}, \\
e^{-\psi_{b}^{-1} \kappa\left(t_{u}-t_{0}\right)}=e^{-2 n c_{\zeta 2} \psi_{b}^{-1} \sqrt{\kappa} \ln \left(\omega_{o} \rho_{0}\right)} \leq \frac{1}{\rho_{0}^{n} \omega_{o}^{n}},
\end{array}\right.
$$

for $\frac{\omega_{o}}{\kappa} \geq 1$ and $\kappa \geq \frac{1}{4 \psi_{b}^{-2} c_{\varsigma 2}^{2}}$. Owing to (40)-(41), we have

$$
\left\{\begin{array}{c}
\sqrt{V_{\varsigma}\left(t_{u}\right)} \leq \eta_{V_{\zeta} 1}\left(\omega_{2}\right) \triangleq \frac{2 c_{\varsigma 2}\left\|P_{\zeta}\right\| \pi_{\zeta 0}\left(\eta_{e 1}\right)}{\sqrt{c_{\zeta 1}} \omega_{2}}+\frac{n \sqrt{c_{\zeta 2}}}{\rho_{0} \omega_{2}} \\
\sqrt{V_{u}\left(t_{u}\right)} \leq \eta_{V_{u} 1}\left(\omega_{2}, \kappa_{2}\right) \triangleq \\
\frac{\sqrt{2} \pi_{\delta_{u} 0}\left(\eta_{e 1}\right)}{2 \psi_{b}^{-1} \kappa_{2}}+\frac{\sqrt{2} \pi_{\omega}\left(\omega_{2}\right) c_{\zeta 2}\left\|P_{\zeta}\right\| \pi_{\zeta 0}\left(\eta_{e 1}\right)}{c_{\zeta 1} \psi_{b}^{-1} \omega_{2}} \\
+\frac{\sqrt{V_{u}\left(t_{0}\right)}}{\omega_{2}^{n} \rho_{0}^{n}}+\frac{\sqrt{2} \pi_{\omega}\left(\omega_{2}\right) n \sqrt{c_{\zeta 2}}}{\sqrt{c_{\zeta 1}} \psi_{b}^{-1} \rho_{0} \omega_{2}}
\end{array}\right.
$$


for any $\omega_{o} \geq \omega_{2}, \kappa \geq \kappa_{2}$ and $\frac{\omega_{o}}{\kappa} \geq \tau_{2}$, where $\omega_{2}=\max \left\{\kappa_{2} \tau_{2}, \omega_{1}\right\}, \kappa_{2}=\frac{1}{4 \psi_{b}^{-2} c_{\xi 2}^{2}}$ and $\tau_{2}=\max \left\{1,4 \psi_{b}^{-1} c_{\varsigma 2}\right\}$.

Considering the initial condition satisfying $\rho_{0} \leq \frac{1}{\omega_{o}}$, (23) implies that $t_{u}=t_{0}$. It can be directly proved that $\left\|e\left(t_{u}\right)\right\|, V_{z}\left(z\left(t_{u}\right)\right), \sqrt{V_{\mathcal{S}}\left(t_{u}\right)}$ and $\sqrt{V_{u}\left(t_{u}\right)}$ are bounded for ${ }_{225} \omega_{o} \geq \omega_{2}$. Without loss of generality, the bounds of $\left\|e\left(t_{u}\right)\right\|, V_{z}\left(z\left(t_{u}\right)\right), \sqrt{V_{\varsigma}\left(t_{u}\right)}$ and $\sqrt{V_{u}\left(t_{u}\right)}$ can be also denoted as $\eta_{e 1}, \eta_{V_{z} 1}, \eta_{V_{\varsigma} 1}$ and $\eta_{V_{u} 1}$, respectively.

Part 2: The analysis for the closed-loop system for $t \in\left[t_{u}, \infty\right)$.

According to the control design (19) and the closed-loop system (30)-(31), the variables $(e, \zeta, z, u)$ are continuous at $t_{u}$. To simplify the mathematical expressions in this part, we introduce the following notations.

$$
\left\{\begin{array}{l}
\eta_{V_{K} 2} \triangleq \sqrt{c_{k 2}} \max \left\{\eta_{e 1}, 2\left\|P_{K}\right\| \psi_{b} \eta_{\delta_{u} 2}\right\}, \quad \eta_{V_{\varsigma} 2} \triangleq \eta_{V_{\varsigma} 1}, \\
\eta_{V_{u} 2} \triangleq \max \left\{\eta_{V_{u} 1}, 2 \pi_{\omega}\left(\omega_{2}\right) \eta_{\zeta 2} \psi_{b}\right\}, \quad \eta_{V_{z} 2}=r_{z}\left(n \psi_{\varphi}\left(\eta_{e 2}+M_{x^{*}}\right)\right), \\
\eta_{e 2} \triangleq \frac{\eta_{V_{K} 1}}{\sqrt{c_{k 1}}}, \quad \eta_{\zeta 2} \triangleq \frac{\eta_{V 2}}{\sqrt{c_{21}}}, \quad \eta_{\delta_{u} 2} \triangleq \frac{\eta_{V 2}}{\sqrt{c_{21}}} .
\end{array}\right.
$$

Then we proceed to prove that there exist positive constants $\left(\omega_{3}, \kappa_{3}, \tau_{3}\right)$ such that $\left(e(t), z(t), \zeta(t), \delta_{u}(t)\right)$ stay in a bounded set $\Omega_{1} \triangleq\left\{\left(e, z, \zeta, \delta_{u}\right) \mid \sqrt{V_{K}} \leq \eta_{V_{K}}, \sqrt{V_{z}} \leq\right.$ $\left.\eta_{V_{z} 2}, \sqrt{V_{\varsigma}} \leq \eta_{V_{\varsigma} 2}, \sqrt{V_{u}} \leq \eta_{V_{u} 2}\right\}$ for $\omega_{o} \geq \omega_{3}, \kappa \geq \kappa_{3}, \frac{\omega_{o}}{\kappa} \geq \tau_{3}$ and $t \geq t_{u}$. The proof consists of the following four steps:

Step 1. We assume that there exists $t^{*} \in\left[t_{u}, \infty\right)$ such that $\sqrt{V_{\varsigma}\left(t^{*}\right)}=\eta_{V_{\varsigma} 2}$. Besides, $\sqrt{V_{\varsigma}(t)} \leq \eta_{V_{\varsigma} 2}, \sqrt{V_{K}(t)} \leq \eta_{V_{K} 2}, V_{z}(t) \leq \eta_{V_{z} 2}$ and $\sqrt{V_{\delta_{u}}(t)} \leq \eta_{V_{u} 2}$ for $t \in\left[t_{u}, t^{*}\right]$. Then it can be deduced that $\|e(t)\| \leq \eta_{e 2},\|\zeta(t)\| \leq \eta_{\zeta 2}$ and $\left|\delta_{u}(t)\right| \leq \eta_{\delta_{u} 2}$ for $t \in\left[t_{u}, t^{*}\right]$. Owing to the dynamics (31) and the bound of $\Delta_{\zeta 1}$ (32), the derivative of $\sqrt{V_{\zeta}\left(t^{*}\right)}$ satisfies

$$
\frac{d \sqrt{V_{\zeta}\left(t^{*}\right)}}{d t} \leq-\frac{\omega_{o} \eta_{V_{\varsigma} 2}}{2 c_{\zeta 2}}+\frac{\left\|P_{\varsigma}\right\|\left(\pi_{\zeta 1}\left(\eta_{e 2}\right)+(\kappa+1) \pi_{\delta_{u}}\left(\eta_{e 2}, \eta_{\delta_{u} 2}\right)+\pi_{\omega}\left(\omega_{o}\right) \kappa \eta_{\zeta 2}\right)}{\sqrt{c_{\zeta 1}}}
$$

By selecting $\omega_{3}=\max \left\{\omega_{2},\left(6 c_{\varsigma 2}\left\|P_{\varsigma}\right\|\left(\pi_{\zeta 1}\left(\eta_{e 2}\right)+\pi_{\delta_{u}}\left(\eta_{e 2}, \eta_{\delta_{u} 2}\right)\right)\right) /\left(\eta_{V_{\varsigma} 2} \sqrt{c_{\zeta 1}}\right)\right\}$ and $\tau_{3}=\left(6 c_{\zeta 2}\left\|P_{\varsigma}\right\|\left(\pi_{\omega}\left(\omega_{2}\right) \eta_{\zeta 2}+\pi_{\delta_{u}}\left(\eta_{e 2}, \eta_{\delta_{u} 2}\right)\right)\right) /\left(\eta_{V_{\varsigma} 2 \sqrt{c_{\zeta 1}}}\right)$, (43) directly implies that $\frac{d \sqrt{V_{\varsigma}\left(t^{*}\right)}}{d t}<0$ for the $\left(\omega_{o}, \kappa\right)$ satisfying $\omega_{o} \geq \omega_{3}$ and $\frac{\omega_{o}}{\kappa} \geq \tau_{3}$.

Step 2. We assume that there exists $t^{*} \in\left[t_{u}, \infty\right)$ such that $V_{z}\left(t^{*}\right)=\eta_{V_{z}}$. Besides, $\sqrt{V_{u}(t)} \leq \eta_{V_{u} 2}, \sqrt{V_{K}(t)} \leq \eta_{V_{K} 2}, V_{z}(t) \leq \eta_{V_{z} 2}$ and $\sqrt{V_{\varsigma}(t)} \leq \eta_{V_{\varsigma} 2}$ for $t \in\left[t_{u}, t^{*}\right]$. From 
Lemma 1, the bound of $x(t)$ for $t \in\left[t_{u}, t^{*}\right]$ satisfies that

$$
\sup _{t_{u} \leq t \leq t^{*}}\|x(t)\| \leq n \psi_{\varphi}\left(\sup _{t_{u} \leq t \leq t^{*}}\|\tilde{x}(t)\|\right) \leq n \psi_{\varphi}\left(\eta_{e 2}+M_{x^{*}}\right) .
$$

The definition of $\eta_{V_{z} 2}$ (42) implies that $\eta_{V_{z} 2} \geq r_{z}\left(\sup _{t_{u} \leq t \leq t^{*}}\|x(t)\|\right) \geq r_{z}\left(\left\|x\left(t^{*}\right)\right\|\right)$. Due to Assumption 3, it can be verified that $\dot{V}_{z}\left(z\left(t^{*}\right)\right) \leq 0$.

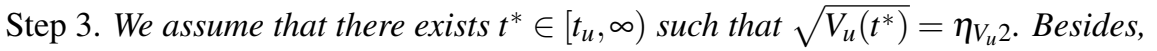
$\sqrt{V_{u}(t)} \leq \eta_{V_{u} 2}, \sqrt{V_{K}(t)} \leq \eta_{V_{K} 2}, V_{z}(t) \leq \eta_{V_{z} 2}$ and $\sqrt{V_{\varsigma}(t)} \leq \eta_{V_{\varsigma} 2}$ for $t \in\left[t_{u}, t^{*}\right]$. Hence $\|e(t)\| \leq \eta_{e 2},\|\zeta(t)\| \leq \eta_{\zeta 2},\left|\delta_{u}(t)\right| \leq \eta_{\delta_{u} 2}$ and $|b(x, t)| \geq \eta_{\underline{b} 2}$ for $t \in\left[t_{u}, t^{*}\right]$. Based on (31) and (32), the derivative of $\sqrt{V_{u}\left(t^{*}\right)}$ satisfies

$$
\frac{d \sqrt{V_{u}\left(t^{*}\right)}}{d t} \leq-\psi_{b}^{-1} \eta_{V_{u} 2} \kappa+\frac{\sqrt{2}}{2}\left(\pi_{\delta_{u} 1}\left(\eta_{e 2}, \eta_{\delta_{u} 2}\right)+\pi_{\omega} \kappa \eta_{\zeta 2}\right) .
$$

Owing to the definition of $\eta_{V_{u} 2}$ (42), we have $-\frac{\psi_{b}^{-1} \eta_{V_{u} 2}}{2}+\pi_{\omega}\left(\omega_{2}\right) \eta_{\zeta 2}=-\pi_{\omega}\left(\omega_{2}\right) \eta_{\zeta 2} \leq$ 0 for any $\omega_{o} \geq \omega_{3}$. By choosing $\kappa_{3}=\frac{2 \sqrt{2} \pi_{\delta_{u} 1}\left(\eta_{e 2}, \eta_{\delta_{u} 2}\right)}{\eta_{b 2} \eta_{V_{u} 2}}$, there is

$$
-\frac{\psi_{b}^{-1} \eta_{V_{u} 2}}{2} \kappa+\frac{\sqrt{2} \pi_{\delta_{u} 1}\left(\eta_{e 2}, \eta_{\delta_{u} 2}\right)}{2}<0
$$

for $\kappa \geq \kappa_{3}$. Hence $\frac{d \sqrt{V_{u}\left(t^{*}\right)}}{d t}<0$ for $\omega_{o} \geq \omega_{3}$ and $\kappa \geq \kappa_{3}$.

Step 4. We assume that there exists $t^{*} \in\left[t_{u}, \infty\right)$ such that $\sqrt{V_{K}\left(t^{*}\right)}=\eta_{V_{K} 2}$. Besides, $\sqrt{V_{K}(t)} \leq \eta_{V_{K} 2}, \sqrt{V_{\varsigma}(t)} \leq \eta_{V_{\varsigma} 2}, V_{z}(t) \leq \eta_{V_{z} 2}$ and $\sqrt{V_{u}(t)} \leq \eta_{V_{u} 2}$ for $t \in\left[t_{u}, t^{*}\right]$. Hence $\|e(t)\| \leq \eta_{e 2},\|\zeta(t)\| \leq \eta_{\zeta 2}$ and $\left|\delta_{u}(t)\right| \leq \eta_{\delta_{u} 2}$ for $t \in\left[t_{u}, t^{*}\right]$. According to (31), (32) and (42), the derivative of $\sqrt{V_{K}\left(t^{*}\right)}$ satisfies

$$
\frac{d \sqrt{V_{K}\left(t^{*}\right)}}{d t} \leq-\frac{\left\|e\left(t^{*}\right)\right\|}{2 \sqrt{V_{K}\left(t^{*}\right)}}\left(\left\|e\left(t^{*}\right)\right\|-2\left\|P_{K}\right\| \psi_{b} \eta_{\delta_{u} 2}\right)<0 .
$$

Due to Steps $1-4$, it can be concluded that the variables $\left(e(t), z(t), \zeta(t), \delta_{u}(t)\right)$ stay in $\Omega_{1}$ for $t \in\left[t_{u}, \infty\right)$ if the parameters satisfy $\omega_{o} \geq \omega_{3}, \kappa \geq \kappa_{3}$ and $\frac{\omega_{o}}{\kappa} \geq \tau_{3}$. Next, we will carefully analyze the bounds of $\sqrt{V_{K}(t)}, \sqrt{V_{\varsigma}(t)}$ and $\sqrt{V_{u}(t)}$ for $t \geq t_{u}$.

The analysis of the bound of $\sqrt{V_{\varsigma}(t)}$. Due to (31) and (32), for $t \geq t_{u}$, we have $\frac{d \sqrt{V_{\zeta}(t)}}{d t} \leq-\frac{\omega_{o}}{2 c_{\zeta 2}} \sqrt{V_{\varsigma}(t)}+\frac{\left\|P_{\zeta}\right\|\left(\pi_{\zeta 1}\left(\eta_{e 2}\right)+(\kappa+1) \pi_{\delta_{u}}\left(\eta_{e 2}, \eta_{\delta_{u} 2}\right)+\pi_{\omega}\left(\omega_{o}\right) \kappa \eta_{\zeta 2}\right)}{\sqrt{c_{\zeta 1}}}$. 
Combined with Gronwall lemma, $\sqrt{V_{\varsigma}(t)}$ has the following bound.

$$
\sqrt{V_{\varsigma}(t)} \leq \eta_{V_{\varsigma} 2} e^{-\frac{\omega_{o}\left(t-t_{u}\right)}{2 c_{\varsigma 2}}}+\theta_{\varsigma 1} \frac{1}{\omega_{o}}+\theta_{\varsigma^{2}} \frac{\kappa}{\omega_{o}}, \quad \forall t \in\left[t_{u}, \infty\right)
$$

for $\omega_{o} \geq \omega_{3}, \kappa \geq \kappa_{3}$ and $\frac{\omega_{o}}{\kappa} \geq \tau_{3}$, where $\theta_{\varsigma 1}=\left(\left\|P_{\zeta}\right\|\left(\pi_{\zeta 1}\left(\eta_{e 2}\right)+\pi_{\delta_{u}}\left(\eta_{e 2}, \eta_{\delta_{u} 2}\right)\right) / \sqrt{c_{\zeta 1}}\right.$ and $\theta_{\varsigma 2}=\left(\left\|P_{\varsigma}\right\|\left(\pi_{\delta_{u}}\left(\eta_{e 2}, \eta_{\delta_{u} 2}\right)+\pi_{\omega}\left(\omega_{3}\right) \eta_{\zeta 2}\right)\right) / \sqrt{c_{\varsigma 1}}$.

The analysis of the bound of $\sqrt{V_{u}(t)}$. Owing to (31), (32) and (47), for $t \geq t_{u}$, there is

$$
\begin{aligned}
\frac{d \sqrt{V_{u}(t)}}{d t} \leq & -\psi_{b}^{-1} \kappa \sqrt{V_{u}(t)}+\frac{\sqrt{2}}{2}\left(\pi_{\delta_{u} 1}\left(\eta_{e 2}, \eta_{\delta_{u} 2}\right)+\pi_{\omega} \kappa\|\zeta(t)\|\right) \\
\leq & -\psi_{b}^{-1} \kappa \sqrt{V_{u}(t)} \\
& +\frac{\sqrt{2}}{2}\left(\pi_{\delta_{u} 1}\left(\eta_{e 2}, \eta_{\delta_{u} 2}\right)+\kappa \frac{\pi_{\omega}\left(\eta_{V_{\varsigma} 2} e^{-\frac{\omega_{o}}{2 c_{\zeta 2}}\left(t-t_{u}\right)}+\theta_{\zeta 1} \frac{1}{\omega_{o}}+\theta_{\zeta 2} \frac{\kappa}{\omega_{o}}\right)}{\sqrt{c_{\zeta 1}}}\right) .
\end{aligned}
$$

Combined with Gronwall lemma, the bound of $\sqrt{V_{u}(t)}$ is shown as follows.

$$
\begin{aligned}
\sqrt{V_{u}(t)} & \leq \eta_{V_{u} 2} e^{-\psi_{b}^{-1} \kappa\left(t-t_{u}\right)}+\frac{\theta_{u 1}}{\omega_{o}}+\frac{\theta_{u 2} \kappa}{\omega_{o}}+\frac{\theta_{u 3}}{\kappa}+\theta_{u 4} \int_{t_{u}}^{t} e^{-\psi_{b}^{-1} \kappa(t-s)} e^{-\frac{\omega_{o}}{2 c_{\varsigma 2}}\left(s-t_{u}\right)} d s \\
& \leq\left(\eta_{V_{u} 2}+\frac{\theta_{u 4}}{\psi_{b}^{-1} \kappa_{3}}\right) e^{-\psi_{b}^{-1} \kappa\left(t-t_{u}\right)}+\theta_{u 1} \frac{1}{\omega_{o}}+\theta_{u 2} \frac{\kappa}{\omega_{o}}+\theta_{u 3} \frac{1}{\kappa}, \quad \forall t \in\left[t_{u}, \infty\right)
\end{aligned}
$$

for $\omega_{o} \geq \omega_{3}, \kappa \geq \kappa_{3}$ and $\frac{\omega_{o}}{\kappa} \geq \tau_{4} \triangleq \max \left\{\tau_{3}, 4 c_{\varsigma 2} \psi_{b}^{-1}\right\}$, where $\theta_{u 1}=\frac{\sqrt{2} \pi_{\omega}\left(\omega_{3}\right) \theta_{\varsigma_{1}}}{2 \psi_{b}^{-1} \sqrt{c_{\varsigma 1}}}, \theta_{u 2}=$ $\frac{\sqrt{2} \pi_{\omega}\left(\omega_{3}\right) \theta_{\zeta^{2}}}{2 \psi_{b}^{-1} \sqrt{c_{\zeta 1}}}, \theta_{u 3}=\frac{\sqrt{2} \pi_{\delta_{u} 1}\left(\eta_{e 2}, \eta_{\delta_{u} 2}\right)}{2 \psi_{b}^{-1}}$ and $\theta_{u 4}=\frac{\sqrt{2} \pi_{\omega}\left(\omega_{3}\right) \eta_{V_{\zeta} 2}}{2 \psi_{b}^{-1} \sqrt{c_{\varsigma} 1}}$.

The analysis of the bound of $\sqrt{V_{K}(t)}$. By denoting $\tilde{t}_{u}=t_{u}+\frac{\ln \omega_{o}}{\psi_{b}^{-1} \sqrt{\kappa}}$, it can be deduced from (31) and (38) that

$$
\left\{\begin{array}{l}
\sup _{t_{u} \leq t \leq \tilde{t}_{u}} \sqrt{V_{K}(t)} \leq \sqrt{c_{k 2}}\left(\left\|e\left(t_{u}\right)\right\|+\left(\left\|A_{K}\right\| \eta_{e 2}+\psi_{b} \eta_{\delta_{u} 2}\right) \frac{\ln \omega_{o}}{\psi_{b}^{-1} \sqrt{\kappa}}\right) \leq \theta_{e} \frac{\ln \omega_{o}}{\sqrt{\kappa}} \\
e^{-\psi_{b}^{-1} \kappa\left(\tilde{t}_{u}-t_{u}\right)}=\frac{1}{\omega_{o}^{\sqrt{\kappa}}} \leq \frac{1}{\omega_{o}}
\end{array}\right.
$$

for $\omega_{o} \geq \omega_{3}, \kappa \geq \kappa_{4} \triangleq \max \left\{\kappa_{3}, 1\right\}$ and $\frac{\omega_{o}}{\kappa} \geq \tau_{4}$, where $\theta_{e}=4 \sqrt{c_{k 2}} n c_{\varsigma 2}\left(\|A\| \eta_{e 1}+\right.$ $\left.\pi_{e 0}\left(\eta_{e 1}\right)\right)+\frac{\sqrt{c_{k 2}}\left\|A_{K}\right\| \eta_{e 2}+\sqrt{c_{k 2}} \psi_{b} \eta_{\delta_{u} 2}}{\psi_{b}^{-1}}$. Then the bound of $\sqrt{V_{K}(t)}$ for $t \geq \tilde{t}_{u}$ is analyzed. According to (31), (32) and (48)-(49), the dynamics of $\sqrt{V_{K}(t)}$ satisfies the following 
equation for $t \geq \tilde{t}_{u}$.

$$
\begin{aligned}
\frac{d \sqrt{V_{K}(t)}}{d t} & \leq-\frac{\sqrt{V_{K}(t)}}{2 c_{k 2}}+\frac{\left\|P_{K}\right\| \psi_{b}\left|\delta_{u}(t)\right|}{\sqrt{c_{k 1}}} \\
& \leq-\frac{\sqrt{V_{K}(t)}}{2 c_{k 2}}+\frac{\sqrt{2}\left\|P_{K}\right\| \psi_{b}}{\sqrt{c_{k 1}}}\left(\left(\eta_{V_{u} 2}+\frac{\theta_{u 4}}{\psi_{b}^{-1} \kappa}\right) e^{-\psi_{b}^{-1} \kappa\left(t-t_{u}\right)}+\frac{\theta_{u 1}}{\omega_{o}}+\frac{\theta_{u 2} \kappa}{\omega_{o}}+\frac{\theta_{u 3}}{\kappa}\right) \\
& \leq-\frac{\sqrt{V_{K}(t)}}{2 c_{k 2}}+\frac{\sqrt{2}\left\|P_{K}\right\| \psi_{b}}{\sqrt{c_{k 1}}}\left(\left(\eta_{V_{u} 2}+\frac{\theta_{u 4}}{\psi_{b}^{-1} \kappa}+\theta_{u 1}\right) \frac{1}{\omega_{o}}+\frac{\theta_{u 2} \kappa}{\omega_{o}}+\frac{\theta_{u 3}}{\kappa}\right)
\end{aligned}
$$

for $\omega_{o} \geq \omega_{3}, \kappa \geq \kappa_{4}$ and $\frac{\omega_{o}}{\kappa} \geq \tau_{4}$. With the help of Gronwall lemma, we get the following bound of $\sqrt{V_{K}}$.

$$
\sup _{t \geq \tilde{t}_{u}} \sqrt{V_{K}(t)} \leq \sqrt{V_{K}\left(\tilde{t}_{u}\right)}+\frac{\theta_{e 1}}{\omega_{o}}+\frac{\theta_{e 2} \kappa}{\omega_{o}}+\frac{\theta_{e 3}}{\kappa} \leq \theta_{e} \frac{\ln \omega_{o}}{\sqrt{\kappa}}+\frac{\theta_{e 1}}{\omega_{o}}+\frac{\theta_{e 2} \kappa}{\omega_{o}}+\frac{\theta_{e 3}}{\kappa},
$$

for $\omega_{o} \geq \omega_{3}, \kappa \geq \kappa_{4}$, and $\frac{\omega_{o}}{\kappa} \geq \tau_{4}$, where $\theta_{e 1}=\frac{2 \sqrt{2} c_{k_{2}}\left\|P_{K}\right\| \psi_{b}}{\sqrt{c_{k 1}}}\left(\eta_{V_{u} 2}+\frac{\theta_{u 4}}{\psi_{b}^{-1} \kappa_{3}}+\theta_{u 1}\right)$, $\theta_{e 2}=\frac{2 \sqrt{2} c_{k 2}\left\|P_{K}\right\| \psi_{b}}{\sqrt{c_{k 1}}} \theta_{u 2}$ and $\theta_{e 3}=\frac{2 \sqrt{2} c_{k 2}\left\|P_{K}\right\| \psi_{b}}{\sqrt{c_{k 1}}} \theta_{u 3}$.

According to Assumption 4, there exists a positive constant $\omega_{4} \geq \omega_{3}$ such that

$$
\kappa\left(\omega_{o}\right) \geq \kappa_{4}, \quad \frac{\omega_{o}}{\kappa\left(\omega_{o}\right)} \geq \tau_{4},
$$

for any $\omega_{o} \geq \omega_{4}$. Notice that $\frac{1}{\omega_{o}} \leq \frac{\kappa}{\omega_{o}}$ for $\kappa \geq \kappa_{4} \geq 1$. With the combination of the bounds of $\sup _{t_{0} \leq t \leq t_{u}}\|e(t)\|, \sqrt{V_{K}(t)}, \sqrt{V_{\varsigma}(t)}$ and $\sqrt{V_{u}(t)}$, i.e., (38), (47), (48), (49) and (50), we prove (24)-(26).

\section{Simulation}

In this section, the simulation for an application example, Chua's circuit, is presented.

Figure 1 describes Chua's circuit, which is featured with strong nonlinearity and can generate chaotic response [36]. The mathematical model of Chua's circuit is presented as follows [32, 36].

$$
\left\{\begin{array}{l}
\dot{x}_{1}(t)=-\frac{1}{C_{1} R} x_{1}(t)+\frac{1}{C_{1} R} x_{2}(t)-\frac{1}{C_{1}} f\left(x_{1}\right), \\
\dot{x}_{2}(t)=-\frac{1}{C_{2} R} x_{1}(t)+\frac{1}{C_{2} R} x_{2}(t)+\frac{1}{C_{2}} x_{3}(t), \\
\dot{x}_{3}(t)=\frac{1}{L} u(t)-\frac{1}{L} x_{2}(t)-\frac{R_{0}}{L} x_{3}(t),
\end{array}\right.
$$




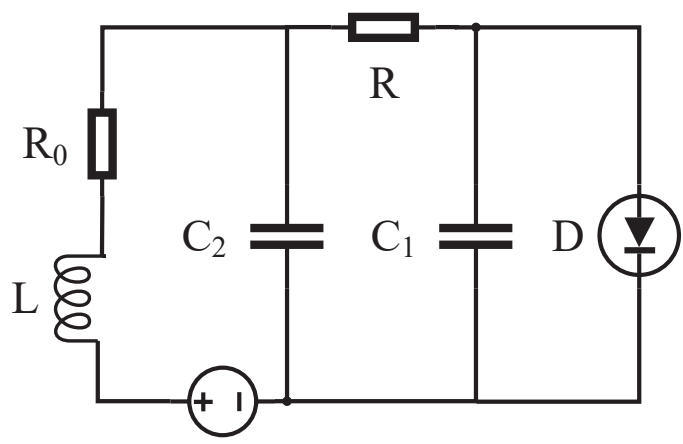

$\mathrm{u}$

Figure 1: Chua's circuit.

where $x_{1}$ and $x_{2}$ represent the voltages across the capacitor $C_{1}$ and $C_{2}, x_{3}$ is the current through the inductor $L, u$ is the input voltage, and $f\left(x_{1}\right)$ represents the nonlinear current caused by the nonlinear resistor $D$.

The control objective is to design the input voltage $u(t)$ such that the system states $\left(x_{1}, x_{2}, x_{3}\right)$ can track $(0,0,0)$.

In the simulation, the measurement of $x_{1}$ can be obtained, while $\left(x_{2}, x_{3}\right)$ cannot be measured. Besides, the detailed values of the system parameters $\left(C_{1}, C_{2}, L, R, R_{0}\right)$ are unknown for the control design, whereas the signs of $\left(C_{1}, C_{2}, L, R, R_{0}\right)$ can be directly verified by physical mechanism:

$$
C_{1}>0, \quad C_{2}>0, \quad L>0, \quad R>0, \quad R_{0} \geq 0 .
$$

According to $[32,36]$, the nonlinearity $f\left(x_{1}\right)$ satisfies that $f(0)=0$.

Remark 7. The presented control objective is a classical stabilization problem [32, 36]. However, the methods proposed in [32, 36] require the measurements for all states $\left(x_{1}, x_{2}, x_{3}\right)$. In this paper, only the measurement of $x_{1}$ is utilized for the stabilization problem. Moreover, the nominal values of system parameters $\left(C_{1}, C_{2}, L, R, R_{0}\right)$ are unknown. 

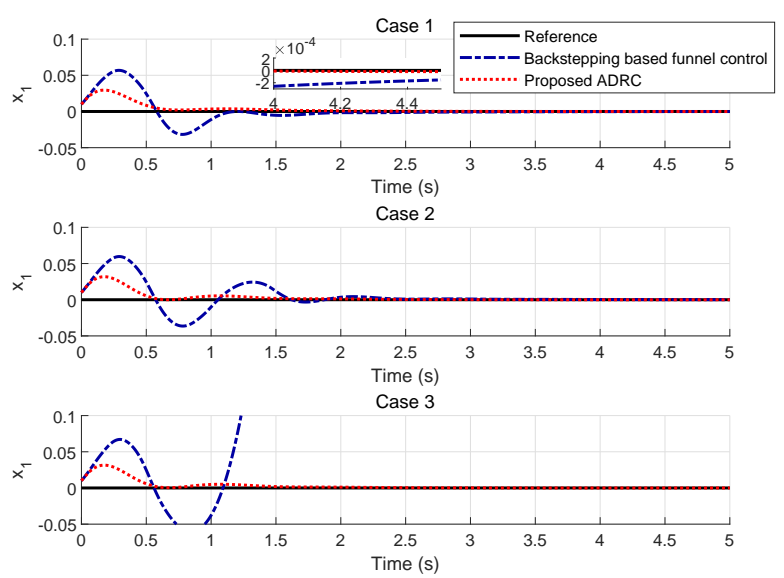

Figure 2: The response curves of the state $x_{1}$.
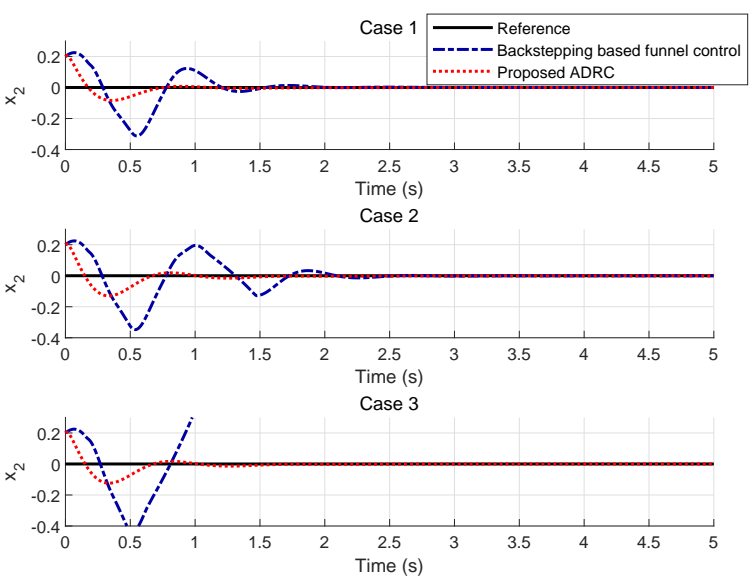

Figure 3: The response curves of the state $x_{2}$.

Next, the proposed ADRC is applied to the system (52). Firstly, denote the new 

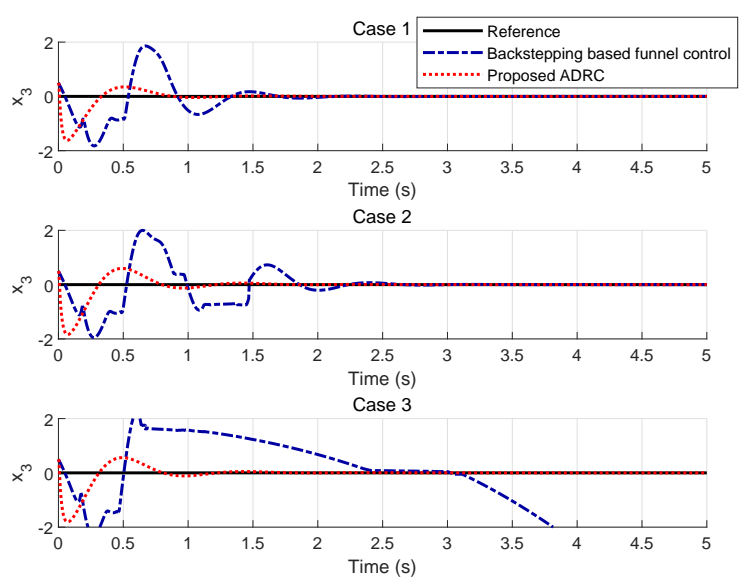

Figure 4: The response curves of the state $x_{3}$.

states

$$
\left\{\begin{aligned}
\tilde{x}_{1}= & x_{1} \\
\tilde{x}_{2}= & -\frac{1}{C_{1} R} x_{1}(t)+\frac{1}{C_{1} R} x_{2}(t)-\frac{1}{C_{1}} f\left(x_{1}\right) \\
\tilde{x}_{3}= & \left(-\frac{1}{C_{1} R}-\frac{1}{C_{1}} \frac{d f\left(x_{1}\right)}{d x_{1}}\right)\left(-\frac{x_{1}(t)}{C_{1} R}+\frac{x_{2}(t)}{C_{1} R}-\frac{f\left(x_{1}\right)}{C_{1}}\right) \\
& +\frac{1}{C_{1} R}\left(-\frac{x_{1}(t)}{C_{2} R}+\frac{x_{2}(t)}{C_{2} R}+\frac{x_{3}(t)}{C_{2}}\right) .
\end{aligned}\right.
$$

Then the integrators chain form is obtained as follows.

$$
\left\{\begin{array}{l}
\dot{\tilde{x}}_{1}(t)=\tilde{x}_{2}(t), \\
\dot{\tilde{x}}_{2}(t)=\tilde{x}_{3}(t), \\
\dot{\tilde{x}}_{3}(t)=b u(t)+f_{t},
\end{array}\right.
$$


where $b$ and $f$ satisfy the following equation.

$$
\left\{\begin{aligned}
b= & \frac{1}{C_{1} C_{2} R L}, \\
f_{t}= & -\frac{1}{C_{1}} \frac{d^{2} f\left(x_{1}\right)}{d x_{1}^{2}}\left(-\frac{x_{1}(t)}{C_{1} R}+\frac{x_{2}(t)}{C_{1} R}-\frac{f\left(x_{1}\right)}{C_{1}}\right)^{2}-\frac{1}{C_{1} C_{2} R^{2}}\left(-\frac{x_{1}(t)}{C_{1} R}+\frac{x_{2}(t)}{C_{1} R}-\frac{f\left(x_{1}\right)}{C_{1}}\right) \\
& +\frac{1}{C_{1} C_{2} R^{2}}\left(-\frac{x_{1}(t)}{C_{2} R}+\frac{x_{2}(t)}{C_{2} R}+\frac{x_{3}(t)}{C_{2}}\right)-\frac{1}{C_{1} C_{2} R}\left(\frac{x_{2}(t)}{L}+\frac{R_{0} x_{3}(t)}{L}\right) \\
& +\left(-\frac{1}{C_{1} R}-\frac{1}{C_{1}} \frac{d f\left(x_{1}\right)}{d x_{1}}\right)\left(-\frac{1}{C_{1} R}-\frac{1}{C_{1}} \frac{d f\left(x_{1}\right)}{d x_{1}}\right)\left(-\frac{x_{1}(t)}{C_{1} R}+\frac{x_{2}(t)}{C_{1} R}-\frac{f\left(x_{1}\right)}{C_{1}}\right) \\
& +\frac{1}{C_{1} R}\left(-\frac{1}{C_{1} R}-\frac{1}{C_{1}} \frac{d f\left(x_{1}\right)}{d x_{1}}\right)\left(-\frac{x_{1}(t)}{C_{2} R}+\frac{x_{2}(t)}{C_{2} R}+\frac{x_{3}(t)}{C_{2}}\right) .
\end{aligned}\right.
$$

Due to the transformation (54), it can be verified that $\left[\begin{array}{lll}x_{1} & x_{2} & x_{3}\end{array}\right]=\left[\begin{array}{lll}0 & 0 & 0\end{array}\right]$ is equivalent to $\left[\begin{array}{lll}\tilde{x}_{1} & \tilde{x}_{2} & \tilde{x}_{3}\end{array}\right]=\left[\begin{array}{lll}0 & 0 & 0\end{array}\right]$. Hence the stabilization problem of the system (52) can be reformulated as the stabilization problem of the system (55).

Although the nominal value of $b$ is unknown due to the unknown system parameters $\left(C_{1}, C_{2}, L, R, R_{0}\right)$, it can be verified by (53) that $\operatorname{sgn}(b)>0$. Based on the sign of $b$, the proposed ADRC (15) and (19) with the following controller parameters is utilized.

$$
\omega_{o}=300, \quad K=\left[\begin{array}{lll}
8 & 12 & 6
\end{array}\right]^{T}, \quad \kappa\left(\omega_{o}\right)=\sqrt{\omega_{o}} .
$$

To investigate the capability of disturbance rejection, the following cases of uncertainties are considered, including the cubic function in [32] (Case 1).

Case 1: $f\left(x_{1}\right)=-x_{1}+x_{1}^{3}$,

Case 2: $f\left(x_{1}\right)=-1.3 x_{1}+x_{1}^{3}$,

Case 3: $f\left(x_{1}\right)=-x_{1}+x_{1}^{3}-\sin \left(x_{1}\right)$.

We consider the following system parameters and initial condition, which are provided in [32].

$$
C_{1}=C_{2}=1, R_{0}=0, R=1, L=2, x_{1}(0)=0.01, x_{2}(0)=0.2, x_{3}(0)=0.5 \text {. }
$$

The simulation results of the proposed ADRC and the following backstepping 
based funnel control [32] are presented in Figures 2-4.

$$
\left\{\begin{array}{l}
u=7 r_{3} \cos \left(\pi r_{3}\right) z_{3} \\
z_{1}=x_{1} \\
z_{2}=x_{2}-10 r_{1} \cos \left(\pi r_{1}\right) z_{1} \\
z_{3}=x_{3}-7 r_{2} \cos \left(\pi r_{2}\right) z_{2} \\
r_{i}=\frac{1}{1-\left(e^{t}-1\right)^{2} z_{i}^{2}}, \quad i=1,2,3
\end{array}\right.
$$

For Case 1, the satisfied closed-loop performance of the proposed ADRC and the backstepping based funnel control are shown, while the smaller tracking error and the better transient performance of the proposed ADRC are illustrated by Figure 2. From Figures $3-4$, the closed-loop performance of the backstepping based funnel control becomes poor for Cases 2-3. Moreover, despite various uncertainties, the performance of the proposed ADRC is highly consistent with each other, which illuminates the strong capability of the proposed ADRC to handle uncertainties.

\section{Conclusion}

For a class of lower-triangular nonlinear uncertain systems, the paper proposes a new ADRC based on the control directions rather than the nominal values or mathematical expressions of control coefficients. The design ideology can be summarized as the following three parts: 1) By transforming the original states into the states of an integrators chain system, the effects from the control input and uncertainties to the controlled output are clearly shown; 2) Based on the integrators chain form, the ESO is presented to estimate the total disturbance and the integrators chain states; 3) Inspired by the approximative dynamic inversion method, a dynamical system is designed to force the actual control input to track the ideal input. Moreover, by associating the parameter of the dynamical input design with the ESO's parameter, the tuning method of the dynamical input design is explicitly provided. With the consideration of a large scope of mismatched nonlinear uncertainties, the transient performance of the proposed

ADRC is theoretically investigated. Based on the presented theoretical results, the sat- 
isfied tracking and estimating performance can be ensured by suitably enlarging the ESO's parameter.

\section{Appendix}

\subsection{Proof of Lemma 1}

Part I: The analysis of the mapping $\varphi$. With the combination of the dynamics (3) and the explicit form of $\tilde{x}_{i}(1 \leq i \leq n)(7)$, it can be directly verified that $\tilde{x}_{i}=\dot{\tilde{x}}_{i-1}$ for $2 \leq i \leq n$. Then, for the transformation (7), we will prove that the term

$$
\tilde{\tau}_{i} \triangleq \Sigma_{j=1}^{i-2} \frac{d^{j-1}}{d t^{j-1}}\left(\frac{d\left(\Pi_{k=1}^{i-j-1} \theta_{k}(t)\right)}{d t} x_{i-j}\right)+\Sigma_{j=1}^{i-1} \frac{d^{j-1}}{d t^{j-1}}\left(\left(\Pi_{k=0}^{i-j-1} \theta_{k}(t)\right) \phi_{i-j}\right)
$$

is a function dependent on $\left(x_{1}, \cdots, x_{i-1}, t\right)$ for $2 \leq i \leq n$ by mathematical induction in the following three steps.

Step 1 (consider the case that $i=2$ ). It can be obtained that

$$
\tilde{\tau}_{2}=\theta_{0}(t) \phi_{1}\left(x_{1}, t\right)=\phi_{1}\left(x_{1}, t\right) .
$$

Hence $\tilde{\tau}_{2}$ is a function dependent on $\left(x_{1}, t\right)$.

Step 2 (consider the case that $i=k(2 \leq k \leq n-1)$ ). Suppose that $\tilde{\tau}_{k}(2 \leq k \leq n-1)$ is a function dependent on $\left(x_{1}, \cdots, x_{k-1}, t\right)$.

Step 3 (consider the case that $i=k+1(2 \leq k \leq n-1)$ ). Since $\tilde{\tau}_{k}$ is a function dependent on $\left(x_{1}, \cdots, x_{k-1}, t\right)$, the state $\tilde{x}_{k}$ has the following form:

$$
\tilde{x}_{k}=\left(\Pi_{j=1}^{k-1} \theta_{j}(t)\right) x_{i}(t)+\tilde{\tau}_{k}\left(x_{1}, \cdots, x_{k-1}, t\right) .
$$

By taking the derivative of $\tilde{x}_{k}$ and utilizing the dynamics (3), there is

$$
\begin{aligned}
\dot{\tilde{x}}_{k}=\tilde{x}_{k+1}= & \left(\Pi_{j=1}^{k-1} \theta_{j}(t)\right)\left(\theta_{k}(t) x_{k+1}(t)+\phi_{k}\left(x_{1}, \cdots, x_{k}, t\right)\right) \\
& +\frac{d\left(\Pi_{j=1}^{k-1} \theta_{j}(t)\right)}{d t} x_{k}(t)+\frac{\partial \tilde{\tau}_{k}\left(x_{1}, \cdots, x_{k-1}, t\right)}{\partial t} \\
& +\Sigma_{j=1}^{k-1} \frac{\partial \tilde{\tau}_{k}\left(x_{1}, \cdots, x_{k-1}, t\right)}{\partial x_{j}}\left(\theta_{j} x_{j+1}+\phi_{j}\left(x_{1}, \cdots, x_{j}, t\right)\right) .
\end{aligned}
$$

By comparing the form (62) with (7), it can be obtained that

$$
\begin{aligned}
\tilde{\tau}_{k+1}= & \left(\Pi_{j=1}^{k-1} \theta_{j}(t)\right) \phi_{k}\left(x_{1}, \cdots, x_{k}, t\right)+\frac{d\left(\Pi_{j=1}^{k-1} \theta_{j}(t)\right)}{d t} x_{k}(t)+\frac{\partial \tilde{\tau}_{k}}{\partial t} \\
& +\sum_{j=1}^{k-1} \frac{\partial \tilde{\tau}_{k}}{\partial x_{j}}\left(\theta_{j} x_{j+1}+\phi_{j}\left(x_{1}, \cdots, x_{j}, t\right)\right) .
\end{aligned}
$$



$\left(x_{1}, \cdots, x_{k}, t\right)$.

Based on the fact that $\tilde{\tau}_{i}$ is a function dependent on $\left(x_{1}, \cdots, x_{i-1}, t\right)$ for $2 \leq i \leq n$, (7) can be rewritten in the following form.

$$
\left\{\begin{array}{l}
\tilde{x}_{1}=x_{1}, \\
\tilde{x}_{i}=\left(\Pi_{j=1}^{i-1} \theta_{j}(t)\right) x_{i}+\tilde{\tau}_{i}\left(x_{1}, \cdots, x_{i-1}, t\right), \quad 2 \leq i \leq n .
\end{array}\right.
$$

According to Assumption 2, the term $\prod_{j=1}^{i-1} \theta_{j}(t)$ is nonzero for $2 \leq i \leq n$. Then the mapping from $(\tilde{x}, t)$ to $(x, t)$ can be determined by the following recursive way.

From (64), the functions $\varphi_{1}$ and $\varphi_{2}$ can be obtained as follows.

$$
\varphi_{1}(\tilde{x}, t)=\tilde{x}_{1}, \quad \varphi_{2}(\tilde{x}, t)=\frac{\tilde{x}_{2}-\tilde{\tau}_{2}\left(x_{1}, t\right)}{\theta_{1}(t)}=\frac{\tilde{x}_{2}-\tilde{\tau}_{2}\left(\varphi_{1}(\tilde{x}, t), t\right)}{\theta_{1}(t)} .
$$

Suppose that $\varphi_{i}(\tilde{x}, t)$ exists for $2 \leq i \leq k \leq n-1$ being an integer. Owing to (64), we can acquire the function $\varphi_{k+1}(\tilde{x}, t)$ as follows.

$$
\varphi_{k+1}(\tilde{x}, t)=\frac{\tilde{x}_{k+1}-\tilde{\tau}_{k+1}\left(\varphi_{1}(\tilde{x}, t), \cdots, \varphi_{k}(\tilde{x}, t), t\right)}{\Pi_{j=1}^{k} \theta_{j}(t)}, \quad 2 \leq k \leq n-1 .
$$

Additionally, it is obvious that $\varphi_{n+1}(\tilde{x}, t)=t$. Moreover, the inverse of the mapping $\varphi$ can be directly obtained by (64).

Then the bounds of $\varphi_{i}(\tilde{x}, t)(1 \leq i \leq n+1)$ are analyzed. Owing to the dynamics (3), the expression of $\tilde{\tau}_{i}(59)$ implies that $\tilde{\tau}_{i}$ is composed of the finite sums and products of $x_{j}(1 \leq j \leq i-1), \phi_{j}(1 \leq j \leq i-1)$, the partial derivatives of $\phi_{j}(1 \leq j \leq i-1)$ up to $(i-j-1)$-th order and $\theta_{j}^{(p)}(1 \leq j \leq i-1,0 \leq p \leq i-j-1)$. According to Assumption 2, there exist continuous functions $\tilde{\psi}_{\tau, i}\left(x_{1}, \cdots, x_{i-1}\right)(2 \leq i \leq n)$ such that

$$
\sup _{t \geq t_{0}}\left|\tilde{\tau}_{i}\left(x_{1}, \cdots, x_{i-1}, t\right)\right| \leq \tilde{\Psi}_{\tau, i}\left(x_{1}, \cdots, x_{i-1}\right), \quad \forall\left[x_{1} \cdots x_{i-1}\right] \in R^{i-1}, 2 \leq i \leq n .
$$

By introducing the non-decreasing continuous function

$$
\psi_{\tau, i}\left(\rho_{x}\right) \triangleq \sup _{\left\|\left[x_{1} \cdots x_{i-1}\right]\right\| \leq \rho_{x}} \tilde{\psi}_{\tau, i}\left(x_{1}, \cdots, x_{i-1}\right)
$$

for $\rho_{x} \geq 0$, (67) implies that

$$
\sup _{t \geq t_{0},\left\|\left[x_{1} \cdots x_{i-1}\right]\right\| \leq \rho_{x}}\left|\tilde{\tau}_{i}\left(x_{1}, \cdots, x_{i-1}, t\right)\right| \leq \psi_{\tau, i}\left(\rho_{x}\right), \quad \forall \rho_{x} \geq 0,2 \leq i \leq n .
$$


Next, the bounds of $\varphi_{i}$ are presented by recursive method. Denote $\tilde{\rho}_{x}$ as a non-negative constant. Based on (65), the bounds of $\varphi_{1}$ and $\varphi_{2}$ are shown as follows.

$$
\left\{\begin{aligned}
\sup _{t \geq t_{0},\|\tilde{\tilde{x}}\| \leq \tilde{\rho}_{x}}\left|\varphi_{1}(\tilde{x}, t)\right| & \leq \sup _{\|\tilde{x}\| \leq \tilde{\rho}_{x}}\left|\tilde{x}_{1}\right| \leq M_{\varphi, 1}\left(\tilde{\rho}_{x}\right) \triangleq \tilde{\rho}_{x}, \\
\sup _{t \geq t_{0},\|\tilde{x}\| \leq \tilde{\rho}_{x}}\left|\varphi_{2}(\tilde{x}, t)\right| & \leq \sup _{t \geq t_{0},\|\tilde{x}\| \leq \tilde{\rho}_{x}} \frac{\left|\tilde{x}_{2}\right|+\left|\tilde{\tau}_{2}\left(\varphi_{1}(\tilde{x}, t), t\right)\right|}{\left|\theta_{1}(t)\right|} \\
& \leq M_{\varphi, 2}\left(\tilde{\rho}_{x}\right) \triangleq \frac{\tilde{\rho}_{x}+\psi_{\tau, 2}\left(M_{\varphi, 1}\left(\tilde{\rho}_{x}\right)\right)}{\underline{M}_{\theta, 1}} .
\end{aligned}\right.
$$

Notice that $M_{\varphi, 1}(\cdot)$ and $M_{\varphi, 2}(\cdot)$ are increasing functions. Suppose that $\sup _{t \geq t_{0},\|\tilde{x}\| \leq \tilde{\rho}_{x}}\left|\varphi_{i}(\tilde{x}, t)\right| \leq$ $M_{\varphi, i}\left(\tilde{\rho}_{x}\right)$ where $M_{\varphi, i}$ is a continuous increasing functions for $2 \leq i \leq k, 2 \leq k \leq n-1$ and $\tilde{\rho}_{x} \geq 0$. Then, the bound of $\varphi_{k+1}(\tilde{x}, t)$ can be obtained as follows.

$$
\begin{aligned}
\sup _{t \geq t_{0},\|\tilde{x}\| \leq \tilde{\rho}_{x}}\left|\varphi_{k+1}(\tilde{x}, t)\right| & \leq \sup _{t \geq t_{0},\|\tilde{x}\| \leq \tilde{\rho}_{x}} \frac{\left|\tilde{x}_{k+1}\right|+\left|\tilde{\tau}_{k+1}\left(\varphi_{1}(\tilde{x}, t), \cdots, \varphi_{k}(\tilde{x}, t), t\right)\right|}{\left|\prod_{j=1}^{k} \theta_{j}(t)\right|} \\
& \leq M_{\varphi, k+1}\left(\tilde{\rho}_{x}\right) \triangleq \frac{\tilde{\rho}_{x}+\psi_{\tau, k+1}\left(\sqrt{\sum_{j=1}^{k}\left(M_{\varphi, j}\left(\tilde{\rho}_{x}\right)\right)^{2}}\right)}{\prod_{j=1}^{k} \underline{M}_{\theta, j}} .
\end{aligned}
$$

Hence it can be concluded that there exist continuous increasing functions $M_{\varphi, i}(1 \leq$ $i \leq n)$ such that $\sup _{t \geq t_{0},\|\tilde{x}\| \leq \tilde{\rho}_{x}}\left|\varphi_{i}(\tilde{x}, t)\right| \leq M_{\varphi, i}\left(\tilde{\rho}_{x}\right)$ for $\tilde{\rho}_{x} \geq 0$ and $1 \leq i \leq n$.

By taking the partial derivatives of $\varphi_{i}$ along the dynamics (3), it can be deduced with the similar analysis (65)-(70) that there exist continuous functions $M_{\frac{\partial \varphi}{\partial \check{x}}, i}$ and $M_{\frac{\partial \varphi}{\partial t}, i}$ such that

$$
\sup _{t \geq t_{0},\|\tilde{x}\| \leq \tilde{\rho}_{x}}\left\|\frac{\partial \varphi_{i}(\tilde{x}, t)}{\partial \tilde{x}}\right\| \leq M_{\frac{\partial \varphi}{\partial \tilde{x}}, i}\left(\tilde{\rho}_{x}\right), \sup _{t \geq t_{0},\|\tilde{x}\| \leq \tilde{\rho}_{x}}\left|\frac{\partial \varphi_{i}(\tilde{x}, t)}{\partial t}\right| \leq M_{\frac{\partial \varphi}{\partial t}, i}\left(\tilde{\rho}_{x}\right), \quad 1 \leq i \leq n .
$$

for any $\tilde{\rho}_{x} \geq 0$. Due to (69)-(71), (10) is proved by defining

$$
\psi_{\varphi}\left(\tilde{\rho}_{x}\right) \triangleq \max _{1 \leq i \leq n}\left\{M_{\varphi, i}\left(\tilde{\rho}_{x}\right), M_{\frac{\partial \varphi}{\partial \tilde{x}}, i}\left(\tilde{\rho}_{x}\right) M_{\frac{\partial \varphi}{\partial t}, i}\left(\tilde{\rho}_{x}\right)\right\} .
$$

Part II: The analysis of the mapping $\gamma$. Based on the analysis of $\tilde{\tau}_{i}$, the mapping $\gamma$ can be directly determined by (64). Combined with the bounds of $\tilde{\tau}_{i}(67)-(68),(64)$ 
further implies that

$$
\left\{\begin{aligned}
& \sup _{t \geq t_{0},\|x\| \leq \rho_{x}}\left|\gamma_{1}(x, t)\right| \leq\left|x_{1}\right| \leq M_{\gamma, 1}\left(\rho_{x}\right) \triangleq \rho_{x} \\
& \sup _{t \geq t_{0},\|x\| \leq \rho_{x}}\left|\gamma_{i}(x, t)\right| \leq \sup _{t \geq t_{0},\|x\| \leq \rho_{x}}\left(\left|\Pi_{j=1}^{i-1} \theta_{j}(t)\right|\left|x_{i}\right|+\left|\tilde{\tau}_{i}\right|\right) \\
& \leq M_{\gamma, i}\left(\rho_{x}\right) \triangleq \rho_{x} \Pi_{j=1} \bar{M}_{\theta, i}+\psi_{\tau, i}\left(\rho_{x}\right), \quad 2 \leq i \leq n
\end{aligned}\right.
$$

for any given $\rho_{x} \geq 0$. Notice that $M_{\gamma, i}\left(\rho_{x}\right)(1 \leq i \leq n)$ are continuous increasing functions.

Similarly, by taking the partial derivatives of $\gamma_{i}$ along the dynamics (3), it can be deduced by Assumption 2 that there exist continuous functions $M_{\frac{\partial \gamma}{\partial x}, i}$ and $M_{\frac{\partial \gamma}{\partial t}, i}$ such that

$$
\sup _{t \geq t_{0},\|x\| \leq \rho_{x}}\left\|\frac{\partial \gamma_{i}(x, t)}{\partial x}\right\| \leq M_{\frac{\partial \gamma}{\partial x}, i}\left(\rho_{x}\right), \sup _{t \geq t_{0},\|x\| \leq \rho_{x}}\left|\frac{\partial \gamma_{i}(x, t)}{\partial t}\right| \leq M_{\frac{\partial \gamma}{\partial t}, i}\left(\rho_{x}\right), \forall 1 \leq i \leq n,
$$

for any $\rho_{x} \geq 0$. Based on (72)-(73) and the notation

$$
\psi_{\gamma}\left(\rho_{x}\right) \triangleq \max _{1 \leq i \leq n}\left\{M_{\gamma, i}\left(\rho_{x}\right), M_{\frac{\partial \gamma}{\partial x}, i}\left(\rho_{x}\right) M_{\frac{\partial \gamma}{\partial t}, i}\left(\rho_{x}\right)\right\}
$$

then (9) is proved.

\subsection{Proof of Lemma 2}

Firstly, the bounds of $b(t), b^{-1}(t)$ and $\dot{b}(t)$ are analyzed. According to the bounds of $\theta_{i}$ and $\dot{\theta}_{i}$ in Assumption 2, the expression of $b(t)$ (12) implies that

$$
\left\{\begin{array}{l}
|b(t)| \leq \Pi_{i=1}^{n} \bar{M}_{\theta, i}, \\
\left|b^{-1}(t)\right|=\left|\Pi_{i=1}^{n} \theta_{i}^{-1}(t)\right| \leq \Pi_{i=1}^{n} \underline{M}_{\theta, i}^{-1} \\
|\dot{b}(t)|=\sum_{j=1}^{n}\left|\dot{\theta}_{j}(t)\right| \Pi_{1 \leq i \leq n, i \neq j}\left|\theta_{i}(t)\right| \leq \prod_{i=1}^{n} \bar{M}_{\theta, i}
\end{array}\right.
$$

By denoting $\psi_{b}=\max \left\{\Pi_{i=1}^{n} \bar{M}_{\theta, i}, \Pi_{i=1}^{n} \underline{M}_{\theta, i}^{-1}\right\},(27)$ is proved.

Next the bound of $f(\tilde{x}, t)$ is analyzed. Lemma 1 illustrates that $\varphi_{n+1-j}(\tilde{x}, t)=$ $x_{n+1-j}$ for $1 \leq j \leq n-1$. Additionally, $\tilde{\phi}_{i}(\tilde{x}, t)=\phi_{i}\left(x_{1}, \cdots, x_{i}, t\right)$ for $1 \leq i \leq n$. From the expression of $f(\tilde{x}, t)(12), f(\tilde{x}, t)$ can be regarded as a function of $(x, t)$, denoted as $\bar{f}(x, t)$. 
Owing to the dynamics (3) and the notation $\bar{f}(x, t)$, it can be verified that $\bar{f}(x, t)$ is composed of the finite sums and products of $x_{j}(1 \leq j \leq n), \phi_{j}(1 \leq j \leq n)$, the partial derivatives of $\phi_{j}(1 \leq j \leq n)$ up to $(n-j)$-th order and $\theta_{j}^{(p)}(1 \leq j \leq n-1,0 \leq p \leq$ $n-j-1)$. Due to Assumption 2, there exists a continuous function $\psi_{f, 1}(x)$ such that

$$
\sup _{t \geq t_{0}}|\bar{f}(x, t)| \leq \psi_{f, 1}(x)
$$

which further implies that

$$
\sup _{t \geq t_{0},\|\tilde{x}\| \leq \tilde{\rho}_{x}}|f(\tilde{x}, t)|=\sup _{t \geq t_{0},\|\tilde{x}\| \leq \tilde{\rho}_{x}}|\bar{f}(x, t)| \leq \sup _{\|\tilde{x}\| \leq \tilde{\rho}_{x}} \psi_{f, 1}(x), \quad \forall \tilde{\rho}_{x} \geq 0 .
$$

Recalling the continuous increasing function $\psi_{\gamma}(\cdot)$ in Lemma 1, owing to inverse function theorem, the inverse function of $\psi_{\gamma}(\cdot)$ can be denoted as $\psi_{\gamma}^{-1}(\cdot)$ which is a continuous function in $[0, \infty)$. According to (8)-(9) in Lemma 1, the following equation holds.

$$
\begin{aligned}
& \sup _{\|x\| \leq \psi_{\gamma}^{-1}\left(\tilde{\rho}_{x} / \sqrt{n}\right)}\|\tilde{x}\|=\sup _{\|x\| \leq \psi_{\gamma}^{-1}\left(\tilde{\rho}_{x} / \sqrt{n}\right)}\left\|\left[\begin{array}{lll}
\gamma_{1}(x, t) & \cdots & \gamma_{n}(x, t)
\end{array}\right]\right\| \\
& \leq \sqrt{n} \psi_{\gamma}\left(\psi_{\gamma}^{-1}\left(\tilde{\rho}_{x} / \sqrt{n}\right)\right)=\tilde{\rho}_{x} .
\end{aligned}
$$

Based on (77), it can be verified that

$$
\left\{\tilde{x} \mid\|\tilde{x}\| \leq \tilde{\rho}_{x}\right\} \subset\left\{\tilde{x} \mid\|x\| \leq \psi_{\gamma}^{-1}\left(\tilde{\rho}_{x} / \sqrt{n}\right)\right\}, \quad \forall \tilde{\rho}_{x} \geq 0
$$

With the combination of (76) and (78), it can be obtained that

$$
\sup _{t \geq t_{0},\|\tilde{x}\| \leq \tilde{\rho}_{x}}|f(\tilde{x}, t)| \leq \sup _{\|\tilde{x}\| \leq \tilde{\rho}_{x}} \psi_{f, 1}(x) \leq \bar{\psi}_{f, 1}\left(\tilde{\rho}_{x}\right) \triangleq \sup _{\|x\| \leq \psi_{\gamma}^{-1}\left(\tilde{\rho}_{x} / \sqrt{n}\right)} \psi_{f, 1}(x), \quad \forall \tilde{\rho}_{x} \geq 0 .
$$

Due to the continuity of $\psi_{\gamma}^{-1}(\cdot)$ and $\psi_{f, 1}(\cdot)$, it can be deduced that $\bar{\psi}_{f, 1}\left(\tilde{\rho}_{x}\right)$ is a continuous function with respect to the variable $\tilde{\rho}_{x}$.

From Lemma $1, \varphi_{n+1}(\tilde{x}, t)=t$ and

$$
x_{i}=\varphi_{i}(\tilde{x}, t), \quad 1 \leq i \leq n .
$$


Then the partial derivatives of $f(\tilde{x}, t)$ can be expressed as follows.

$$
\left\{\begin{aligned}
\frac{\partial f(\tilde{x}, t)}{\partial \tilde{x}_{i}} & =\frac{\partial \bar{f}\left(\varphi_{1}(\tilde{x}, t), \cdots, \varphi_{n}(\tilde{x}, t), t\right)}{\partial \tilde{x}_{i}} \\
& =\Sigma_{j=1}^{n} \frac{\partial \bar{f}\left(x_{1}, \cdots, x_{n}, t\right)}{\partial x_{j}} \frac{\partial \varphi_{j}(\tilde{x}, t)}{\partial \tilde{x}_{i}}, \quad 1 \leq i \leq n, \\
\frac{\partial f(\tilde{x}, t)}{\partial t} & =\frac{\partial \bar{f}\left(x_{1}, \cdots, x_{n}, t\right)}{\partial t} .
\end{aligned}\right.
$$

Due to Assumption 2, with the similar derivation as (75), there exist continuous functions $\psi_{f x, j}$ and $\psi_{f t}$ such that

$$
\sup _{t \geq t_{0}}\left|\frac{\partial \bar{f}\left(x_{1}, \cdots, x_{n}, t\right)}{\partial t}\right| \leq \psi_{f t}(x), \sup _{t \geq t_{0}}\left|\frac{\partial \bar{f}\left(x_{1}, \cdots, x_{n}, t\right)}{\partial x_{j}}\right| \leq \psi_{f x, j}(x), \quad 1 \leq j \leq n .
$$

Based on the bounds of $\frac{\partial \varphi_{j}}{\partial x_{i}}(10)$ and with the similar procedure as (75)-(79), there exist continuous functions $\bar{\psi}_{f, 2}(\cdot)$ and $\bar{\psi}_{f, 3}(\cdot)$ such that

$$
\sup _{t \geq t_{0},\|\tilde{x}\| \leq \tilde{\rho}_{x}}\left\|\frac{\partial f(\tilde{x}, t)}{\partial \tilde{x}}\right\| \leq \bar{\psi}_{f, 2}\left(\tilde{\rho}_{x}\right), \quad \sup _{t \geq t_{0},\|\tilde{x}\| \leq \tilde{\rho}_{x}}\left|\frac{\partial f(\tilde{x}, t)}{\partial t}\right| \leq \bar{\psi}_{f, 3}\left(\tilde{\rho}_{x}\right), \quad \forall \tilde{\rho}_{x} \geq 0 .
$$

By denoting $\psi_{f}\left(\tilde{\rho}_{x}\right) \triangleq \max \left\{\bar{\psi}_{f, 1}\left(\tilde{\rho}_{x}\right), \bar{\psi}_{f, 2}\left(\tilde{\rho}_{x}\right), \bar{\psi}_{f, 3}\left(\tilde{\rho}_{x}\right)\right\}$, (79) and (83) imply (28).

\subsection{Proof of Lemma 3}

For $t \in\left[t_{0}, t_{u}\right)$, the control input satisfies that $u(t)=0$. Hence the dynamics of $\tilde{x}$ in (11) can be rewritten as follows.

$$
\dot{\tilde{x}}(t)=A \tilde{x}(t)+B f(\tilde{x}, t) .
$$

Combined with the dynamics of $\tilde{x}^{*}(17)$, the dynamics of $e(t)$ for $t \in\left[t_{0}, t_{u}\right)$ is shown as follows.

$$
\dot{e}(t)=A e(t)+B \Delta_{e 0}(e, t), \quad t \in\left[t_{0}, t_{u}\right),
$$

where

$$
\Delta_{e 0}(e, t)=f\left(\tilde{x}^{*}+e, t\right)+K^{T}\left(\tilde{x}^{*}(t)-\bar{r}(t)\right)-r^{(n)}(t) .
$$

Then, consider the case that $t \in\left[t_{u}, \infty\right)$. With the notation (1) and the ideal input (18), the dynamics of $\tilde{x}$ in (11) can be reformulated as follows.

$$
\begin{aligned}
\dot{\tilde{x}}(t) & =A \tilde{x}(t)+B\left(b(t) u(t)+f(\tilde{x}, t)-b(x t) u^{*}(t)+b(t) u^{*}(t)\right) \\
& =A \tilde{x}(t)-B K^{T}(\tilde{x}(t)-\bar{r}(t))+B r^{(n)}(t)+B b(t) \delta_{u}(t) \\
& =A_{K} e(t)+B b(t) \delta_{u}(t)+\dot{\tilde{x}}^{*}(t) .
\end{aligned}
$$


Owing to the dynamics of $\tilde{x}^{*}(17)$, the dynamics of $e(t)$ for $t \in\left[t_{u}, \infty\right)$ is obtained as follows.

$$
\dot{e}(t)=A_{K} e(t)+B b(t) \delta_{u}(t), \quad t \in\left[t_{u}, \infty\right) .
$$

Before analyzing the closed-loop form of $\zeta$ and $\delta_{u}$, the derivative of $u^{*}(t)$ is calculated due to (18).

$$
\left\{\begin{aligned}
\dot{u}^{*}(t) & =-\frac{\dot{b}(t)\left(-f(\tilde{x}, t)-K^{T}(\tilde{x}-\bar{r})+r^{(n)}\right)}{b^{2}(t)} \\
& +\frac{-\frac{\partial f}{\partial \tilde{x}}\left(A\left(e+\tilde{x}^{*}\right)+B f\left(e+\tilde{x}^{*}, t\right)\right)-\frac{\partial f}{\partial t}}{b(t)} \\
& +\frac{-K^{T}\left(A\left(e+\tilde{x}^{*}\right)+B f\left(e+\tilde{x}^{*}, t\right)-\dot{\bar{r}}\right)+r^{(n+1)}}{b(t)}, \quad t \in\left(t_{0}, t_{u}\right), \\
\dot{u}^{*}(t) & =-\frac{\dot{b}(t)\left(-f(\tilde{x}, t)-K^{T}(\tilde{x}-\bar{r})+r^{(n)}\right)}{b^{2}(t)} \\
& +\frac{-\frac{\partial f}{\partial \tilde{x}}\left(A_{K} e+B b \delta_{u}+\dot{\tilde{x}}^{*}\right)-\frac{\partial f}{\partial t}}{b(t)} \\
& +\frac{-K^{T}\left(A_{K} e+B b \delta_{u}+\dot{\tilde{x}}^{*}-\dot{\bar{r}}\right)+r^{(n+1)}}{b(t)}, \quad t \in\left(t_{u}, \infty\right) .
\end{aligned}\right.
$$

With the help of Remark 4, the derivative of $u(t)$ is shown as follows.

$$
\begin{aligned}
\dot{u}(t) & =-\operatorname{sgn}(b(t)) \kappa\left(\omega_{o}\right)\left(\hat{f}_{t}(t)+K^{T}(\hat{\tilde{x}}(t)-\bar{r}(t))-r^{(n)}(t)\right) \\
& =-\kappa|b(t)| \frac{f_{t}(\tilde{x}, u, t)+K^{T}(\tilde{x}(t)-\bar{r}(t))-r^{(n)}(t)-K_{e}^{T} T_{1} \zeta}{b(t)} \\
& =-\kappa|b(t)| \delta_{u}+\operatorname{sgn}(b(t)) \kappa K_{e}^{T} T_{1} \zeta,
\end{aligned}
$$

where $K_{e}=\left[\begin{array}{ll}K^{T} & 1\end{array}\right]^{T}$.

Since $T_{1}^{-1} A_{L} T_{1}=\omega_{o} A_{\zeta}$, the dynamics of $\zeta$ for $t \in\left[t_{0}, t_{u}\right)$ can be obtained by (11) and (15):

$$
\dot{\zeta}(t)=\omega_{o} A_{\zeta} \zeta(t)+B_{f} \Delta_{\zeta 0}(e, t), \quad t \in\left[t_{0}, t_{u}\right),
$$

where

$$
\Delta_{\zeta 0}(e, t)=\left.\frac{d f(\tilde{x}, t)}{d t}\right|_{\text {along (84) }}=\frac{\partial f}{\partial \tilde{x}}\left(A\left(e+\tilde{x}^{*}\right)+B f\left(e+\tilde{x}^{*}, t\right)\right)+\frac{\partial f}{\partial t} .
$$

Owing to (90), the dynamics of $\zeta$ for $t \in\left[t_{u}, \infty\right)$ is presented as follows.

$$
\dot{\zeta}(t)=\omega_{o} A_{\zeta} \zeta(t)+B_{f} \Delta_{\zeta 1}\left(e, \zeta, \delta_{u}, \omega_{o}, \kappa, t\right), \quad t \in\left[t_{0}, t_{u}\right),
$$


where

$$
\begin{aligned}
\Delta_{\zeta 1}\left(e, \zeta, \delta_{u}, \omega_{o}, \kappa, t\right)= & \left.\frac{d(b(t) u(t)+f(\tilde{x}, t))}{d t}\right|_{\text {along }(87)} \\
= & \dot{b} \delta_{u}+\dot{b}\left(-f\left(e+\tilde{x}^{*}, t\right)-K^{T}\left(e+\tilde{x}^{*}-\bar{r}\right)+r^{(n)}\right) / b \\
& +b\left(-\kappa|b| \delta_{u}+\operatorname{sgn}(b) \kappa K_{e}^{T} T_{1} \zeta\right) \\
& +\frac{\partial f}{\partial \tilde{x}}\left(A_{K} e+B b \delta_{u}+\dot{\tilde{x}}^{*}\right)+\frac{\partial f}{\partial t} .
\end{aligned}
$$

With the combination of (89) and (90), the derivative of $\delta_{u}$ is shown as follows.

$$
\left\{\begin{array}{l}
\dot{\delta}_{u}(t)=-|b(t)| \kappa \delta_{u}(t)+\Delta_{\delta_{u} 0}\left(e, \zeta, \omega_{o}, \kappa, t\right), \quad t \in\left[t_{0}, t_{u}\right), \\
\dot{\delta}_{u}(t)=-|b(t)| \kappa \delta_{u}(t)+\Delta_{\delta_{u}}\left(e, \zeta, \delta_{u}, \omega_{o}, \kappa, t\right), \quad t \in\left[t_{u}, \infty\right),
\end{array}\right.
$$

where

$$
\left\{\begin{array}{c}
\Delta_{\delta_{u} 0}\left(e, \zeta, \omega_{o}, \kappa, t\right)=\operatorname{sgn}(b(t)) \kappa K_{e}^{T} T_{1} \zeta+\frac{\dot{b}(t)\left(-f(\tilde{x}, t)-K^{T}\left(e+\tilde{x}^{*}-\bar{r}\right)+r^{(n)}\right)}{b^{2}(t)} \\
-\frac{-\frac{\partial f}{\partial \tilde{x}}\left(A\left(e+\tilde{x}^{*}\right)+B f\left(e+\tilde{x}^{*}, t\right)\right)-\frac{\partial f}{\partial t}-K^{T}\left(A\left(e+\tilde{x}^{*}\right)+B f\left(e+\tilde{x}^{*}, t\right)-\dot{\bar{r}}\right)+r^{(n+1)}}{b(t)} \\
\Delta_{\delta_{u} 1}\left(e, \zeta, \delta_{u}, \omega_{o}, \kappa, t\right)=\operatorname{sgn}(b(t)) \kappa K_{e}^{T} T_{1} \zeta+\frac{\dot{b}(t)\left(-f(\tilde{x}, t)-K^{T}\left(e+\tilde{x}^{*}-\bar{r}\right)+r^{(n)}\right)}{b^{2}(t)} \\
-\frac{-\frac{\partial f}{\partial \tilde{x}}\left(A_{K} e+B b \delta_{u}+\dot{x}^{*}\right)-\frac{\partial f}{\partial t}-K^{T}\left(A_{K} e+B b \delta_{u}+\dot{x}^{*}-\dot{\bar{r}}\right)+r^{(n+1)}}{b(t)}
\end{array}\right.
$$

As for the dynamics of $z$, it can be directly obtained from (11) that

$$
\dot{z}(t)=g\left(z, \varphi_{1}(\tilde{x}, t), \cdots, \varphi_{n}(\tilde{x}, t), t\right), \quad t \geq t_{0} .
$$

Based on the dynamics of $\left(e, \zeta, \delta_{u}, z\right)$, i.e., (85), (88), (91), (93), (95) and (97), the closed-loop form can be written as (30)-(31).

Next we will prove that the bounds of $\left(\Delta_{e 0}, \Delta_{\zeta 0}, \Delta_{\delta_{u} 0}, \Delta_{e 1}, \Delta_{\zeta 1}, \Delta_{\delta_{u} 1}\right)$ satisfy (32) for $e \in\left\{e \mid\|e\| \leq \rho_{e}\right\}, \zeta \in\left\{\zeta \mid\|\zeta\| \leq \rho_{\zeta}\right\}, \delta_{u} \in\left\{\delta_{u}|| \delta_{u} \mid \leq \rho_{u}\right\}$ and $\omega_{o} \in\left\{\omega_{o} \mid \omega_{o} \geq \omega_{o}^{*}\right\}$ with any given positives $\rho_{e}, \rho_{\zeta}, \rho_{u}$ and $\omega_{o}^{*}$. Hence we only need to present the detailed expressions of the functions $\pi_{e 0}(\cdot), \pi_{\zeta 0}(\cdot), \pi_{\zeta 1}(\cdot), \pi_{\delta_{u}}(\cdot), \pi_{\delta_{u} 0}(\cdot)$ and $\pi_{\delta_{u} 1}(\cdot)$.

Due to Assumption 1, there is

$$
\sup _{t \geq t_{0}}\|\bar{r}(t)\| \leq n M_{r}, \quad \sup _{t \geq t_{0}}\left\{\left|r^{(n)}(t)\right|,\left|r^{(n+1)}(t)\right|\right\} \leq M_{r}
$$


Combined with the dynamics of $\tilde{x}^{*}(17)$, there exists a positive constant $M_{x^{*}}$ such that

$$
\sup _{t \geq t_{0}}\left\{\left\|\tilde{x}^{*}(t)\right\|,\left\|\dot{\tilde{x}}^{*}(t)\right\|\right\} \leq M_{x^{*}}
$$

According to the bounds of $\left(b, \dot{b}, b^{-1}, f, \frac{\partial f}{\partial \tilde{x}}, \frac{\partial f}{\partial t}\right)$ shown in Lemma 2, (86) implies that

$$
\sup _{\|e\| \leq \rho_{e}}\left|\Delta_{e 0}(e, t)\right| \leq \pi_{e 0}\left(\rho_{e}\right) \triangleq \psi_{f}\left(\rho_{e}+M_{x^{*}}\right)+\|K\|\left(M_{x^{*}}+n M_{r}\right)+M_{r}, \quad \forall \rho_{e} \geq 0 .
$$

Due to (88), it can be directly obtained that

$$
\left|\Delta_{e 1}\left(\delta_{u}, t\right)\right|=\left|b(t) \delta_{u}(t)\right| \leq \psi_{b}\left|\delta_{u}\right|
$$

Owing to (92) and (94), the bounds of $\Delta_{\zeta 0}$ and $\Delta_{\zeta 1}$ are provided as follows.

$$
\begin{gathered}
\sup _{\|e\| \leq \rho_{e}}\left|\Delta_{\zeta 0}(e, t)\right| \leq \pi_{\zeta 0}\left(\rho_{e}\right) \triangleq \psi_{f}\left(\rho_{e}+M_{x^{*}}\right)\left(1+\|A\|\left(\rho_{e}+M_{x^{*}}\right)+\psi_{f}\left(\rho_{e}+M_{x^{*}}\right)\right), \\
\sup _{\|e\| \leq \rho_{e},\left|\delta_{u}\right| \leq \rho_{u}, \omega_{o} \geq \omega_{o}^{*}}\left|\Delta_{\zeta 1}\left(e, \zeta, \delta_{u}, \omega_{o}, \kappa, t\right)\right| \leq \pi_{\zeta 1}\left(\rho_{e}\right)+\pi_{\omega}\left(\omega_{o}^{*}\right) \kappa\|\zeta\|+(\kappa+1) \pi_{\delta_{u}}\left(\rho_{e}, \rho_{u}\right),
\end{gathered}
$$

for any $\rho_{e} \geq 0, \rho_{u} \geq 0$ and $\omega_{o}^{*} \geq 0$, where $\pi_{\zeta 1}\left(\rho_{e}\right)=\psi_{b}^{2}\left(\psi_{f}\left(\rho_{e}+M_{x^{*}}\right)+\|K\|\left(\rho_{e}+\right.\right.$ $\left.\left.M_{x^{*}}+n M_{r}\right)+M_{r}\right)+\psi_{f}\left(\rho_{e}+M_{x^{*}}\right)\left(1+\left\|A_{K}\right\| \rho_{e}+M_{x^{*}}\right), \pi_{\omega}\left(\omega_{o}^{*}\right)=\max \left\{1, \psi_{b}\right\}\left\|K_{e}\right\|\left\|T_{1}\left(\omega_{o}^{*}\right)\right\|$ and $\pi_{\delta_{u}}\left(\rho_{e}, \rho_{u}\right)=\max \left\{\psi_{b}^{2} \rho_{u}, \psi_{b} \rho_{u}\left(1+\psi_{f}\left(\rho_{e}+M_{x^{*}}\right)\right)\right\}$. With the help of Lemma 2 , the following bounds of $\Delta_{\delta_{u} 1}$ and $\Delta_{\delta_{u}}$ are obtained based on (96).

$$
\left\{\begin{array}{l}
\sup _{\|e\| \leq \rho_{e}}\left|\Delta_{\delta_{u} 0}\right| \leq \pi_{\delta_{u} 0}\left(\rho_{e}\right)+\pi_{\omega}\left(\omega_{o}^{*}\right) \kappa\|\zeta\|, \\
\sup _{\|e\| \leq \rho_{e},\left|\delta_{u}\right| \leq \rho_{u}}\left|\Delta_{\delta_{u} 1}\right| \leq \pi_{\delta_{u} 1}\left(\rho_{e}, \rho_{u}\right)+\pi_{\omega}\left(\omega_{o}^{*}\right) \kappa\|\zeta\|,
\end{array} \quad \forall \rho_{e} \geq 0, \rho_{u} \geq 0\right.
$$

where $\pi_{\delta_{u} 0}\left(\rho_{e}\right)=\max \left\{\psi_{b}, \psi_{b}^{3}\right\}\left(\psi_{f}\left(\rho_{e}+M_{x^{*}}\right)\left(2+\|A\|\left(\rho_{e}+M_{x^{*}}\right)+\psi_{f}\left(\rho_{e}+M_{x^{*}}\right)\right)+\right.$ 330 $\left.\|K\|(1+\|A\|)\left(\rho_{e}+M_{x^{*}}+n M_{r}+\psi_{f}\left(\rho_{e}+M_{x^{*}}\right)\right)+2 M_{r}\right)$ and $\pi_{\delta_{u} 0}\left(\rho_{e}, \rho_{u}\right)=\pi_{\delta_{u} 0}\left(\rho_{e}\right)+$ $\psi_{b}\left(\psi_{f}\left(\rho_{e}+M_{x^{*}}\right)+\|K\|\right)\left(\left\|A_{K}\right\| \rho_{e}+\psi_{b} \rho_{u}+M_{x^{*}}+n M_{r}\right)$.

Based on (100)-(102), (32) is proved.

\section{Data availability statements}

The datasets generated during and/or analysed during the current study are available 


\section{Acknowledgements}

This work was supported in part by the National Natural Science Foundation of China (Nos. 62003202, 61973202 and 61903085), Fundamental Research Funds for the Central Universities (No. GK202003008), Guangdong Basic and Applied Basic Research Foundation (No.2019A1515111070), China Postdoctoral Science Foundation (No.2020M672965) and State Key Laboratory of Synthetical Automation for Process Industries.

\section{Conflict of interest}

The authors declare that they have no conflict of interest regarding the publication of this paper.

\section{References}

[1] K. J. Åström, P. R. Kumar, Control: A perspective, Automatica 50 (1) (2014) 3-43.

[2] C. Zhao, L. Guo, PID controller design for second order nonlinear uncertain systems, Science China Information Sciences 60 (2) (2017) 022201.

[3] M. Benosman, Model-based vs data-driven adaptive control: An overview, International Journal of Adaptive Control and Signal Processing 32 (5) (2018) 753 776.

[4] S. Li, J. Yang, W.-H. Chen, X. Chen, Disturbance observer-based control: Methods and applicaitons, CRC Press, Boca Raton, Fl, 2013.

[5] J. Na, B. Jing, Y. Huang, G. Gao, C. Zhang, Unknown system dynamics estimator for motion control of nonlinear robotic systems, IEEE Transactions on Industrial Electronics 67 (5) (2020) 3850-3859.

[6] P. Selvaraj, O. Kwon, S. Lee, R. Sakthivel, Uncertainty and disturbance rejections of complex dynamical networks via truncated predictive control, Journal of the Franklin Institute 357 (8) (2020) 4901 - 4921. 
[7] J. Han, From PID to active disturbance rejection control, IEEE Transactions on Industrial Electronics 56 (3) (2009) 900-906.

[8] H. Sun, Q. Sun, W. Wu, Z. Chen, J. Tao, Altitude control for flexible wing unmanned aerial vehicle based on active disturbance rejection control and feedforward compensation, International Journal of Robust and Nonlinear Control 30 (1) (2020) 222-245.

[9] J. Tao, Q. Sun, P. Tan, Z. Chen, Y. He, Active disturbance rejection control (adrc)based autonomous homing control of powered parafoils, Nonlinear Dynamics 86 (3) (2016) 1461-1476.

[10] E. Zhu, J. Pang, N. Sun, H. Gao, Q. Sun, Z. Chen, Airship horizontal trajectory tracking control based on active disturbance rejection control, Nonlinear Dynamics 75 (4) (2012) 725-734.

[11] R. Madonski, S. Shao, H. Zhang, Z. Gao, J. Yang, S. Li, General error-based active disturbance rejection control for swift industrial implementations, Control Engineering Practice 84 (2019) 218-229.

[12] W. Wei, W. Xue, D. Li, On disturbance rejection in magnetic levitation, Control Engineering Practice 82 (2019) $24-35$.

[13] L. Sun, J. Shen, Q. Hua, K. Y. Lee, Data-driven oxygen excess ratio control for proton exchange membrane fuel cell, Applied energy 231 (2018) 866-875.

[14] L. Sun, Y. Jin, F. You, Active disturbance rejection temperature control of open-cathode proton exchange membrance fuel cell, Applied energy 261 (2020) 114381.

[15] C. L. Garzon, J. A. Cortes, E. Tello, Active disturbance rejection control for growth of microalgae in a batch culture, IEEE Latin America Transactions 15 (4) (2017) 588-594.

[16] S. Chen, W. Bai, Y. Hu, Y. Huang, Z. Gao, On the conceptualization of total disturbance and its profound implications, Science China Information Sciences 63 (2) (2020) 1-129201. 
[17] B.-Z. Guo, Z.-L. Zhao, On convergence of non-linear extended state observer for multi-input multi-output systems with uncertainty, IET Control Theory \& Applications 6 (15) (2012) 2375-2386.

[18] Q. Zheng, L. Gao, Z. Gao, On stability analysis of active disturbance rejection control for nonlinear time-varying plants with unknow dynamics, 46th IEEE Congerence On Decision and Control, 2007, pp. 3501-3506.

[19] W. Xue, Y. Huang, Performance analysis of active disturbance rejection tracking control for a class of uncertain LTI systems, ISA Transactions 58 (2015) 133-154.

[20] Z.-L. Zhao, B.-Z. Guo, A novel extended state observer for output tracking of mimo systems with mismatched uncertainty, IEEE Transactions on Automatic Control 63 (1) (2017) 211-218.

[21] W. Xue, Y. Huang, Performance analysis of 2-DOF tracking control for a class of nonlinear uncertain systems with discontinuous disturbances, International Journal of Robust and Nonlinear Control 28 (4) (2018) 1456-1473.

[22] C. Aguilar-Ibañez, H. Sira-Ramirez, J.-A. Acosta, Stability of active disturbance rejection control for uncertain systems: A Lyapunov perspective, International Journal of Robust and Nonlinear Control 27 (18) (2017) 4541-4553.

[23] S. Chen, W. Xue, Y. Huang, On active disturbance rejection control for nonlinear systems with multiple uncertainties and nonlinear measurement, International Journal of Robust and Nonlinear Control 30 (8) (2020) 3411-3435.

[24] S. Chen, Z. Chen, On active disturbance rejection control for a class of uncertain systems with measurement uncertainty, IEEE Transactions on Industrial Electronics 68 (2) (2021) 1475-1485.

[25] K. Song, T. Hao, H. Xie, Disturbance rejection control of airfuel ratio with transport-delay in engines, Control Engineering Practice 79 (2018) 36 - 49.

[26] S. Chen, W. Xue, S. Zhong, Y. Huang, On comparison of modified ADRCs for nonlinear uncertain systems with time delay, Science China Information Sciences 61 (7) (2018) 70223. 
[27] W. Bai, W. Xue, Y. Huang, H. Fang, On extended state based kalman filter design for a class of nonlinear time-varying uncertain systems, Science China Information Sciences 61 (4) (2018) 042201.

[28] Z.-H. Wu, B.-Z. Guo, Active disturbance rejection control to MIMO nonlinear systems with stochastic uncertainties: approximate decoupling and outputfeedback stabilisation, International Journal of Control 93 (6) (2020) 1408-1427.

[29] Y. Huang, W. Xue, Active disturbance rejection control: Methodology and theoretical analysis, ISA Transaction 53 (2014) 963-976.

[30] Z. H. Wu, H. C. Zhou, B. Z. Guo, F. Deng, Review and new theoretical perspectives on active disturbance rejection control for uncertain finite-dimensional and infinite-dimensional systems, Nonlinear Dynamics 101 (12).

[31] S. Chen, Y. Huang, Z.-L. Zhao, The necessary and sufficient condition for the uncertain control gain in active disturbance rejection control, https://arxiv.org/abs/2006.11731 (2020). arXiv:2006.11731.

[32] F. Li, Y. Liu, Control design with prescribed performance for nonlinear systems with unknown control directions and nonparametric uncertainties, IEEE Transactions on Automatic Control 63 (10) (2018) 3573-3580.

${ }_{435}$ [33] D. Yoo, S. S.-T. Yau, Z. Gao, Optimal fast tracking observer bandwidth of the linear extended state observer, International Journal of Control 80 (1) (2007) 102111.

[34] N. Hovakimyan, E. Lavretsky, A. Sasane, Dynamic inversion for nonaffine-incontrol systems via time-scale separation. Part I, Journal of Dynamical and Control Systems 13 (2007) 451-465.

[35] M. Ran, Q. Wang, C. Dong, Active disturbance rejection control for uncertain nonaffine-in-control nonlinear systems, IEEE Transactions on Automatic Control 62 (11) (2017) 5830-5836. 
[36] L. Liu, J. Huang, Adaptive robust stabilization of output feedback systems with application to Chua's circuit, IEEE Transactions on Circuits and Systems Part II: Express Briefs 53 (9) (2006) 926-930. 